Research paper

\title{
Peritumoral CD90+CD73+ cells possess immunosuppressive features in human non-small cell lung cancer
}

\author{
Limei Wang $^{\mathrm{a}, \mathrm{b}, 1}$, Haitang Yang ${ }^{\mathrm{a}, \mathrm{b}, 1,2}$, Patrick Dorn ${ }^{\mathrm{a}}$, Sabina Berezowska ${ }^{\mathrm{c}}$, Fabian Blank ${ }^{\mathrm{b}, \mathrm{d}}$, \\ Carlos Wotzkow ${ }^{\mathrm{d}}$, Thomas M. Marti ${ }^{\mathrm{a}, \mathrm{b}}$, Ren-Wang Peng ${ }^{\mathrm{a}, \mathrm{b}}$, Nathalie Harrer ${ }^{\mathrm{e}}$, \\ Wolfgang Sommergruber ${ }^{\mathrm{e}}$, Gregor J. Kocher ${ }^{\mathrm{a}, \mathrm{b}}$, Ralph A. Schmid ${ }^{\mathrm{a}, \mathrm{b}, *}$, Sean R.R. Hall ${ }^{\mathrm{a}, \mathrm{b}, 3, *}$ \\ a Division of General Thoracic Surgery, Bern University Hospital, Bern, Switzerland \\ ${ }^{\mathrm{b}}$ Department of BioMedical Research, University of Bern, Murtenstrasse 50, Bern 3008, Switzerland \\ ${ }^{\mathrm{C}}$ Institute of Pathology, University of Bern, Switzerland \\ ${ }^{\mathrm{d}}$ DCR Live Imaging Core, University of Bern, Switzerland \\ e Boehringer Ingelheim, RCV GmbH \&' Co KG, Vienna, Austria
}

\section{A R T I C L E I N F O}

\section{Article History:}

Received 22 October 2019

Revised 7 September 2021

Accepted 18 October 2021

Available online 2 November 2021

\section{Keywords:}

Stroma

PD-L1

T cells

Immunosuppression

TGF $\beta 1$

Lung cancer

\section{A B S T R A C T}

Background: Although T cell abundance in solid tumours is associated with better outcomes, it also correlates with a stroma-mediated source of immune suppression driven by TGF $\beta 1$ and poor overall survival. Whether this also is observed in non-small cell lung cancer (NSCLC) is unknown.

Methods: We utilized molecular analysis of The Cancer Genome Atlas (TCGA) NSLCC cohort to correlate immune activation (IA) gene expression and extracellular matrix/stromal (ECM/stromal) gene expression with patient survival. In an independent cohort of NSCLC samples, we used flow cytometry to identify mesenchymal subsets and ex vivo functional studies to characterize their immune regulatory function.

Findings: We observed a high enrichment in a core set of genes defining an IA gene expression signature in NSCLC across TCGA Pan-cancer cohort. High IA signature score correlates with enrichment of ECM/stromal gene signature across TCGA NSCLC datasets. Importantly, a higher ratio of ECM/stromal to IA gene signature score was associated with shorter overall survival. In tumours resected from a separate cohort of NSCLC patients, we identified CD90+CD73+ peritumoral cells that were enriched in the ECM/stromal gene signature, which was amplified by TGF $\beta 1$. IFN $\gamma$ and TNF $\alpha$-primed peritumoral CD90+CD73+ cells upregulate immune checkpoint molecules PD-L1 and IDO1 and secrete an array of cytokines/chemokines including TGF $\beta 1$. Finally, immune primed peritumoral CD90+CD73+ cells suppress T cell function, which was relieved following combined blockade of PD-L1 and TGF $\beta 1$ with ID01 inhibition but not PD-L1 or anti-CD73 alone. Interpretation: Our findings suggest that targeting PD-L1 together with independent biological features of the stroma may enhance host antitumor immunity in NSCLC.

Funding: LW and HY are supported by a 4-year China Scholarship Council award. This work was funded, in part, by a grant from the Cancer League of Bern, Switzerland to SRRH. Laser scanning microscopy imaging was funded by the R'Equip grant from the Swiss National Science Foundation Nr. 316030_145003.

(C) 2021 The Authors. Published by Elsevier B.V. This is an open access article under the CC BY license (http://creativecommons.org/licenses/by/4.0/)

\footnotetext{
* Corresponding authors at: Division of General Thoracic Surgery, Bern University Hospital, Bern, Switzerland.

E-mail addresses: Ralph.Schmid@insel.ch (R.A.Schmid), srrhall@protonmail.com (S.R.R. Hall).

1 These authors contributed equally to this work.

2 Present address: Department of Thoracic Surgery, Shanghai Chest Hospital, Shanghai Jiao Tong University, Shanghai 200030, China.

3 Present address: Wyss Institute for Biologically Inspired Engineering, Harvard University, Boston, Massachusetts
}

\section{Introduction}

The introduction of novel therapies aimed at boosting the host immune response against the tumour by blockade of the immune checkpoint molecule programmed cell death receptor 1 (PD-1) or its cognate ligand programmed cell death ligand 1 (PD-L1) has shown durable clinical responses in first and second line setting in a subset of patients with advanced non-small cell lung cancer (NSCLC) [1-5]. Despite this, the majority of advanced NSCLC patients derive no clinical benefit from immune checkpoint blockade (ICB) targeting the PD1/PD-L1 axis [6]. Interferon-gamma (IFN- $\gamma$ ) released by tumour- 


\section{Research in context}

\section{Evidence before this study}

Blockade of immune-checkpoint programmed death 1 (PD-1) or its cognate ligand PD-1 ligand (PD-L1) using antibodies has shown durable antitumor responses only in a minority of patients with advanced non-small cell lung cancer (NSCLC). Why the majority of patients fail to respond is not clearly understood. Emerging evidence in other solid tumor types such as melanoma and urothelial cancer show that $\mathrm{T}$ cell abundance correlates with a stroma-mediated source of immune resistance originating in transforming growth factor beta1 (TGF $\beta 1$ )responsive cancer-associated fibroblasts (CAFs). Importantly, this signature is linked with poor overall survival and resistance to immune checkpoint inhibitors. Whether CAFs or other mesenchymal cell types contribute to a tumour cell-extrinsic resistance signature in NSCLC is not known.

\section{Added value of this study}

We curated a core set of genes defining an immune activated (IA) signature and explored the relationship between this IA signature and ECM/stromal signature expressed by mesenchymal cells within the tumour microenvironment of various solid tumours using molecular analysis of The Cancer Gene Atlas (TCGA) NSCLC dataset. We observed a high enrichment of the IA gene signature in NSCLC and across TCGA Pan-cancer cohort. These immunologically active tumours showed a positive correlation with ECM/stromal gene expression. However, a higher ECM/stromal to IA signature ratio was associated with worse patient survival. To explore the potential source of the ECM/ stromal gene signature in NSCLC, we used multiparametric flow cytometry and were able to identify a subset of nonhematopoietic, non-endothelial cells that were marked by CD90 and CD73 within tumour digests obtained from NSCLC patients undergoing resection for curative intent. These cells overexpress PD-L1, were peritumoral and enriched in the ECM/ stromal gene signature, which was amplified by TGF $\beta 1$. Peritumoral $\mathrm{CD} 90+\mathrm{CD} 73+$ possess immunosuppressive features, unleashed following exposure to pro-inflammatory cytokines TNF $\alpha$ and IFN $\gamma$, potently suppressing T cell function using both cell contact and secreted soluble factors. Combined blockade of PD-L1, TGF $\beta 1$ and indoleamine 2,3-dioxygenase 1 (IDO1) rather than PD-L1 or CD73 blockade alone was able to restore the function of T cells.

\section{Implications of all the available evidence}

Our findings in this study raise the possibility that peritumoral CD90+CD73+ mesenchymal cells may perform an immune sentinel function in highly inflamed tumours and contribute to tumour cell-extrinsic negative regulation of host immunity. Whether this distinct cell subset gives rise to CAFs is not known, as CD90+CD73+ peritumoral cells were also a major source of inflammatory-driven IL-6, which was previously shown in NSCLC to be secreted from CAFs. Second, future preclinical studies using intact organisms will be required to determine whether there is a causal link between targeting the immune suppressive features of the stroma and enhanced antitumor immunity and response to immune checkpoint blockade in NSCLC.

reactive infiltrating lymphocytes (TILs) represents a major driver of tumour cell-extrinsic PD-L1 expression and immune escape. Targets of IFN- $\gamma$ signalling in the tumour microenvironment (TME) release additional molecules such as indoleamine 2,3-dioxygenase 1 (IDO1)
[7] that may serve as an additional brake on chronic T cell activation to restore immune host balance and prevent tissue pathology [8]. In the setting of malignancy, IFN- $\gamma$-driven tumour cell-extrinsic mechanisms may contribute, in part, to immune resistance [9].

NSCLC is divided into adenocarcinoma (LUAD) and squamous cell carcinoma (LUSC) based on their histological features [10]. However, both LUAD and LUSC form heterogeneous, complex organ-like systems, whereby tumour cells interact with and adapt to a dynamic TME composed of an extracellular matrix (ECM) containing a heterogeneous mix of immune cells, lymphatics, blood vessels with their perivascular supporting cells and fibroblasts [11]. The TME is a salient feature of most carcinoma-derived solid tumours, including NSCLC [12]. Cancer-associated fibroblasts (CAFs) that stain positive for fibroblast activation protein- $\alpha$ (FAP) represent the predominant non-hematopoietic mesenchymal cell type of the reactive stroma found in carcinomas [13]. In human lung tumours, the stromal ECM was shown to regulate both the localization and migration of tumour-reactive $\mathrm{T}$ cells thereby influencing antitumor immunity [14]. A comprehensive molecular analysis of melanoma tumours in humans demonstrated a strong positive correlation between the expression of a distinct set of CAF-related genes and T cell infiltration [15]. Although tumours enriched with $\mathrm{T}$ cells are associated with better overall survival [16], a stroma-mediated source of immune resistance originating in transforming growth factor beta 1 (TGF $\beta 1$ )-responsive CAFs is linked with poor overall survival and resistance to ICB targeting PD-1/PD-L1 [17-19]. However, the origin of CAFs in solid tumors is unclear. Lineage tracing in murines showed that pericytes transition to CAFs [20,21]. Whether CAFs or other mesenchymal cell types present in the TME in NSCLC contribute to this tumor cell-extrinsic resistance signature in NSCLC has not been investigated.

Here, we curated a core set of genes defining an immune activation (IA) CD8+ T effector cell signature [22-24] to explore the relationship with a curated ECM/stromal signature expressed by mesenchymal cells, including but not limited to CAFs, within the TME across various solid tumours $[15,17,19]$ using molecular analysis of The Cancer Gene Atlas (TCGA). We use these signatures to demonstrate that enrichment of the IA gene signature in NSCLC and across TCGA Pan-cancer cohort showed a positive correlation with ECM/ stromal gene expression. In particular, a higher $\mathrm{ECM} /$ stromal to IA ratio is associated with worse patient survival in NSCLC. We use freshly resected tumor material from NSCLC patients to show that mesenchymal cells within the TME marked by CD90 and CD73 are enriched in the ECM/stromal gene signature amplified by TGF $\beta 1$ and are potently immunosuppressive. Finally, we demonstrate that combined blockade of signalling ligands PD-L1 and TGF $\beta 1$ together with inhibition of indoleamine 2,3-dioxygenase 1 (IDO1) was able to reverse, in part, the suppressive effects of peritumoral CD90+CD73+ cells on tumor-infiltrating lymphocytes (TILs).

\section{Methods}

\subsection{Study approval and acquisition of tissue samples}

The study was approved by Ethics Commission of the Canton of Bern (KEK-BE:042/2015). All patients gave informed written consent for use of surgical material for research purposes. Lung tumour samples were obtained from patients operated on for NSCLC with a curative intent at Bern University Hospital, Division of General Thoracic Surgery, September 2013 to January 2019. Unfixed surgical specimens were sent to the Institute of Pathology, University of Bern, where a pathologist dissected the tumor and matched non-tumorous lung tissue for further analysis. Cases included in the study were diagnosed as either LUAD $(n=64)$ or LUSC $(n=59)$. Twenty-nine patients received neoadjuvant treatment. Further clinic-pathological characteristics are provided in Table S1 


\subsection{The Cancer Genome Atlas (TCGA) database and establishment of ECM and IIA gene signatures}

To generate an RNA-based metric to score ECM/stroma, we defined a gene set that contributes to the ECM, epithelial-to-mesenchymal transition (EMT) or stroma where there would be overlap in the expression found in the two main mesenchymal cell subsets in solid tumours: cancer-associated fibroblasts (CAFs) and pericytes. We chose hallmark CAF/ pericyte genes VCAN, FAP, COL1A1, POSTN, THY1, FBLN1, and TGF $\beta 1$ mined from three sources $[15,17,19]$. We also choose genes IL6, CSPG4, PDPN, HGF, SERPINE1 specific to lung perivascular-like cells $[25,26]$. The defined gene set for the immune activation (IA) signature was chosen using hallmark genes with cytolytic activity expressed by CD8 TILs (GZMA, GZMB, GZMK, PRF1, IFNG, GNLY and IL2) [22,24] and global activation of CD8 TILs based on single-cell RNA sequencing data in lung cancer patients [23]. Transcriptomic data were obtained from The Cancer Genome Atlas (TCGA) (https://portal.gdc.cancer.gov/projects/TCGA). After normalization (Limma package in the R) and log2 transformation, transcriptomic data were subjected to further analysis. Gene signature sore calculation: after scaling the genes expression value by Apply function in $\mathrm{R}$, a sum of gene expression of the selected genes within the gene signature was then summarised as a single score for each sample. The gene expression and corresponding survival data were extracted for correlation and prognostic analysis using the corresponding packages in $\mathrm{R}$ ('corrplot and Hmisc' packages for correlation analysis; 'maxstat', 'survival' and 'survminer' packages for prognostic analysis).

\subsection{Flow cytometric profiling and prospective cell isolation}

Generation of single-cell suspensions from tumour and matched non-tumorous lung tissue for flow cytometric profiling and prospective cell isolation using fluorescence-activated cell sorting (FACS) have been previously described [27]. Briefly, single cells were stained in buffer with Fc block (eBioscience) containing a panel of fluorescently conjugated human monoclonal antibodies directed at the following epitopes: CD45, CD14, CD31, CD235a into one channel, CD73, CD90, PD-L1, CD47 and EpCAM (see Table S2). To exclude cells from the analysis that stain for lineage markers not expressed by mesenchymal or epithelial cells, we created a dump gate pooling CD45, CD14, CD31, CD235a into one channel (see Fig. S4 for full gating strategy). In a second smaller cohort, single cells were stained with a second antibody panel: CD45, CD14, CD235a, CD31, CD73, CD90, CD39, PD-L1, PDGFR $\alpha$ and EpCAM (see Table S2). To exclude cells from the analysis that stain for lineage markers not expressed by mesenchymal, endothelial or epithelial cells, we created a dump gate pooling CD45, CD14, CD235a into one channel. Flow cytometric profiling of the mesenchymal compartment of single-cell digests of tumour and matched uninvolved lung tissue was performed using a BD FACS LSRII (BD Biosciences). For analysis, a minimum $5 \times 10^{5}$ live events were collected and analyzed using FlowJo software ver10.7.1. PD-L1 and CD47 expression were measured as geometric mean fluorescence intensity (gMFI) in FlowJo. To prospectively isolate mesenchymal cells from the tumour and matched uninvolved tissue, single cells were stained as described above and sorted directly into collection buffer containing 20\% FBS using a BD FACS Aria III or BD FACS Aria. Following this, cells were expanded in $\alpha$-MEM (Sigma) supplemented with $1 \%$ FBS (Invitrogen), $10 \mathrm{ng} / \mathrm{ml}$ of recombinant human bFGF (Gibco, Invitrogen), $20 \mathrm{ng} / \mathrm{ml}$ of recombinant EGF (Gibco, Invitrogen) and $1.25 \mathrm{mg}$ of human insulin solution (Sigma) and 1X antimycotic/antibiotic (Gibco, Invitrogen). Culture expanded cells were used for all downstream experiments (See Supplemental Materials and Methods for details).

\subsection{Statistical analysis}

Data are expressed as mean $\pm \mathrm{SD}$. Comparisons between two groups were carried out using the parametric student's two-tailed paired or unpaired t-test for normally distributed data. If data were not distributed normally, a nonparametric Wilcoxon signed-rank test was used between the two groups. One-way analysis of variance (ANOVA) followed by post hoc Tukey' range test was used for analysis of more than two groups. The numbers of samples (biological replicates) per group ( $\mathrm{n}$ ), or the numbers of experiments (technical replicates) are specified in the figure legends. Data were analyzed using GraphPad Prism 8 software. For survival analysis, patients were grouped by gene expression, where 'high' and 'low' expression groups were stratified by the optimal cutoff value. Then, KaplerMeier analysis of a TCGA cohort of patients with LUSC and LUAD was performed. Stratification of patients into high_extracellular matrix/ stromal (ECM/stromal) (in red) and low_ECM/stromal (in black) or high_IA and low_IA is based on the optimal cutoff value of ECM/stromal gene signature score transcripts across all patients by using the surv_cutpoint function in $\mathrm{R}$ 'maxstat' package. Overall survival curves and cumulative hazard rates were analyzed and plotted by using $\mathrm{R}$ 'survival' and 'survminer' packages. The p-value is calculated using the log-rank test. All other statistical analyses were performed in the R Statistical Computing environment v3.3.1 (http://www.r-project. org). Statistical significance is accepted at $\mathrm{p}<0.05$.

\subsubsection{Role of funding source}

The Funders had no role in the study design, data collection, data analyses, interpretation, or writing of report, and the decision of paper submission.

\section{Results}

\subsection{ECM/stromal gene signature is correlated with PD-L1 expression and CD8 T effector cell phenotypic gene signature in NSCLC}

Expression of PD-L1 represents an adaptive response to proinflammatory molecules IFN $\gamma$ and TNF $\alpha$ released from tumour-reactive effector T cells [28] and represents a predictive biomarker for ICB in NSCLC; although this remains controversial [29]. As recently shown, IFN $\gamma$-driven gene signatures may represent a better prognostic indicator for overall patient survival $[22,30]$. To investigate this in NSCLC, we generated a 13-gene CD8+ T effector cell phenotypic immune activation (IA) signature consisting of a core set of cytotoxic effector molecules (IFN- $\gamma$, GMZA, GZMB, GZMK, PRF1, GNLY), markers of activation (IL-2, PDCD1 (PD-1), CD274 (PD-L1), and CTLA4) together with infiltration (CXCR3) and homeostatic regulation (IL7R or CD127). The genes within the defined IA signature show a mutually significant positive correlation in TCGA LUAD and LUSC datasets (Fig. 1a), indicating a similar immunoregulatory pattern among genes in the set. We also observed a high enrichment in the IA signature score in LUAD and LUSC samples across TCGA Pan-cancer cohort, suggesting an enriched CD8+ T effector cell phenotype (Fig. 1b). Previous studies have shown a positive correlation between $\mathrm{T}$ cell abundance and epithelial-to-mesenchyme (EMT) and stromal-specific gene expression profile originating in non-tumour stroma cells rather than cancer cells in melanoma and urothelial cancer [15,19]. To explore this correlation in NSCLC, we defined a distinct ECM/stromal transcriptional signature consisting of 12 genes known to be expressed by mesenchymal cells within the TME. These 12 genes were part of a larger TGF $\beta 1$-driven gene signature in CAFs that highly correlated with $\mathrm{T}$ cell infiltration $[15,17,19]$. Within this core signature, we show a mutually significant positive correlation across LUAD and LUSC cohorts (Fig. 1c). In addition, ECM/stromal gene signature scores were enriched in LUAD and LUSC samples across TCGA Pan-cancer cohort (Fig. 1d). Next, we validated a positive correlation between $\mathrm{ECM} /$ stromal gene signature score and IA signature score across TCGA LUAD and LUSC dataset (Fig. 1e), which is independent of tumour stages (Fig. S1a,b). High ECM/stromal gene expression alone was not associated with worse patient survival in LUAD, which was 

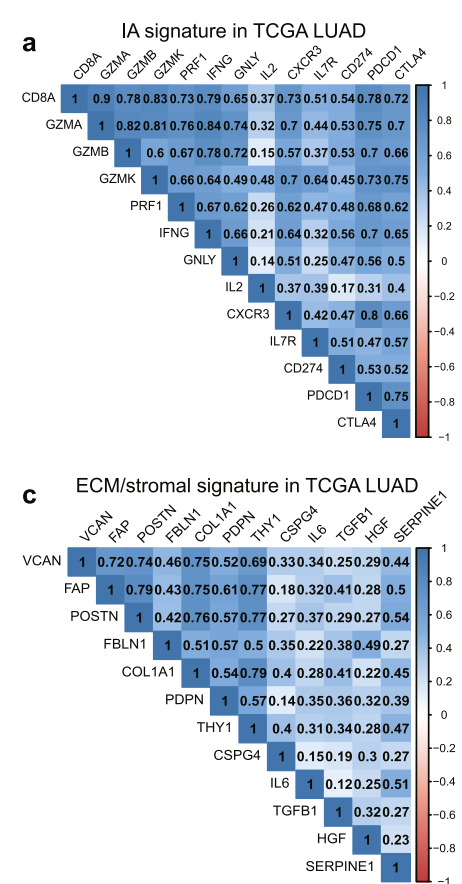

e

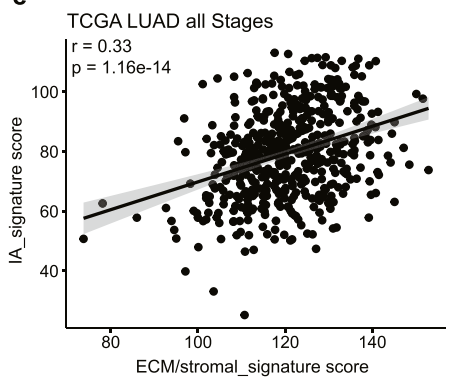

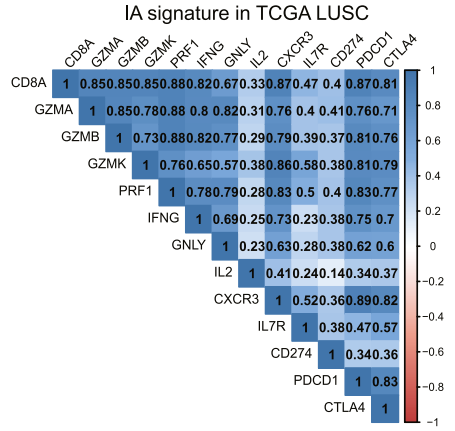
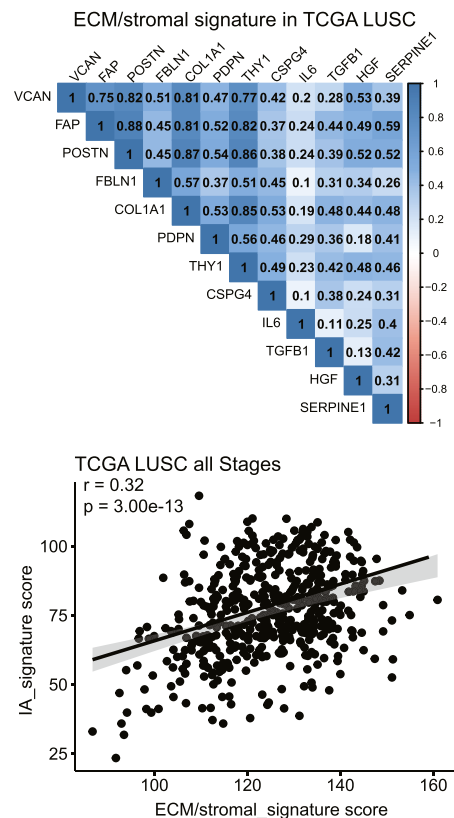

b

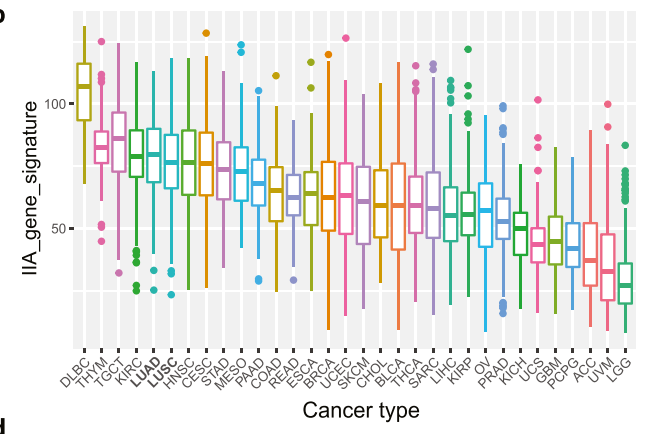

d

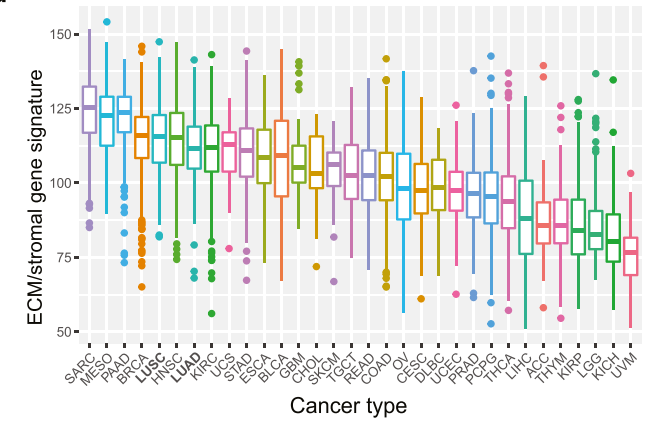

f

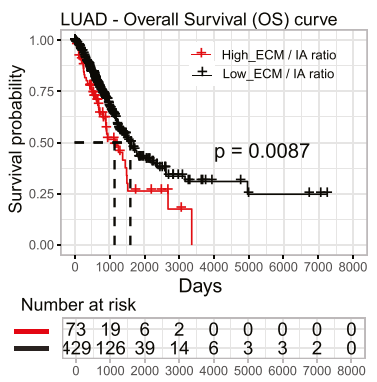

g

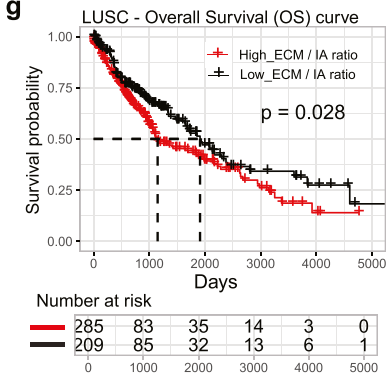

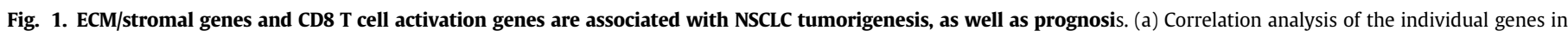

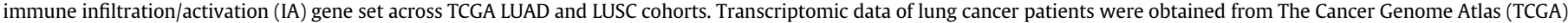

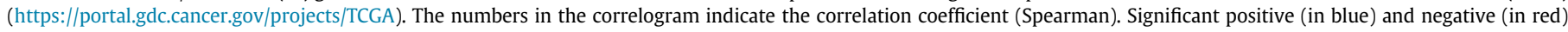

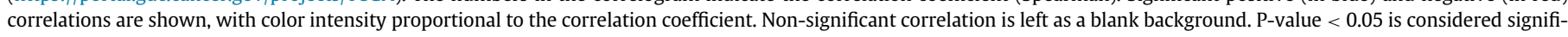

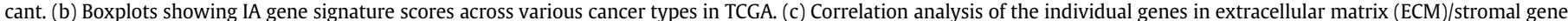

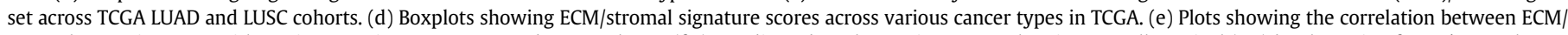

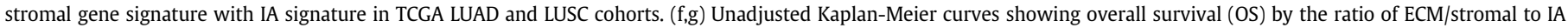
gene expression in LUAD (f) and LUSC ( $g$ ).

in contrast with LUSC where a high ECM/stromal gene score was associated with worse survival (Fig. S1c,d). For both LUAD and LUSC, a high IA gene expression signature score alone was associated with longer patient survival (Fig. S1e,f). When we performed survival analysis using a ratio of ECM/stromal and IA gene signature scores, we observed that a higher ratio of ECM/stromal to IA gene signature score was highly associated with shorter survival in TCGA LUAD and LUSC cohorts (Fig. 1f,g). Collectively, these analyses suggest that the ECM/stromal-defined TME might represent a critical tumour cellextrinsic regulator of tumour immunity in NSCLC.

\subsection{Peritumoral CD90 and CD73 mesenchymal cells are associated with the ECM/stromal gene-set and immunologically active tumors}

Seminal observations from the PanCan Atlas consortium of over 33 cancer types, including LUAD and LUSC, suggest that both the spatial distribution and immune cell composition are determined largely by the cellular makeup of the TME [31,32]. We have previously identified a subset of mesenchymal cells co-expressing thymocyte differentiation antigen 1 (THY1, encoding CD90) and the ecto-5'nucleotidase (NT5E, encoding CD73) with an abnormal perivascularlike function that also expressed PD-L1 in human NSCLC [27]. We validated a high correlation between CD90 mRNA level and the ECM/ stromal signature score across TCGA LUAD and LUSC cohorts (Fig. 2a). CD73 mRNA expression also positively correlates with the ECM/stromal signature score but to a weaker extent (Fig. 2b). A similar trend was also observed between CD90 and CD73 expression with IA gene signature score (Fig. S2a,b). Finally, no correlation between CD90 and CD73 in LUAD (Spearman's $\rho, \mathrm{p}=0.06$ ) was observed, in contrast to LUSC (Spearman's $\rho, \mathrm{p}=0.41$, Fig. 2c). This may indicate a spatial distribution between CD90 and CD73 marked stromal cell populations. Based on univariate analysis, high gene expression of CD90 was associated with worse overall survival in LUSC $(p=0.011)$ but not in LUAD ( $p=0.11$ ) (Fig. S2c), whereas high expression of CD73 was associated with worse patient survival in both $\operatorname{LUAD}(\mathrm{p}=0.0051)$ and $\operatorname{LUSC}(\mathrm{p}=6 \mathrm{e}-04)$ (Fig. S2d).

Next, we examined the spatial distribution of CD90 and CD73 in relationship with tumour epithelium. Confocal imaging showed CD90 and CD73 positive cells marking different mesenchymal cell populations surrounding epithelial cell adhesion molecule (EPCAM) positive tumour islands (Figs. 2d and S2e). Although primarily described as being expressed by tumour or immune cells, PD-L1 expression can be observed in the tumour stroma (Fig. 2e). Overall, there was a strong correlation in PD-L1 at the protein and mRNA level 


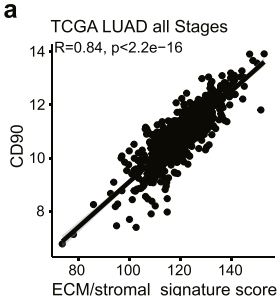

TCGA LUSC all Stages
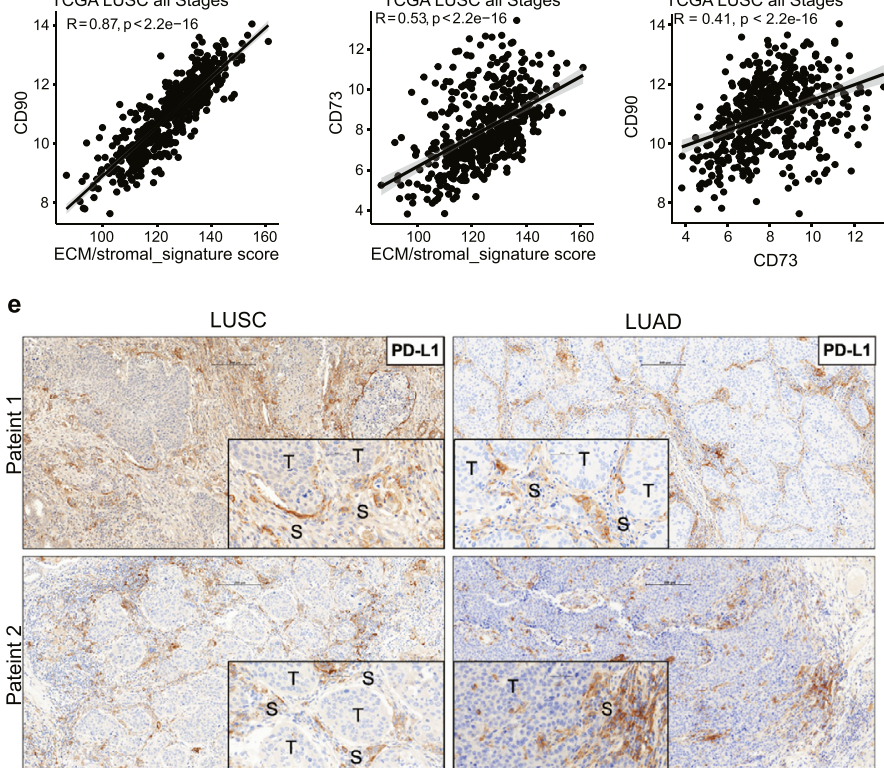
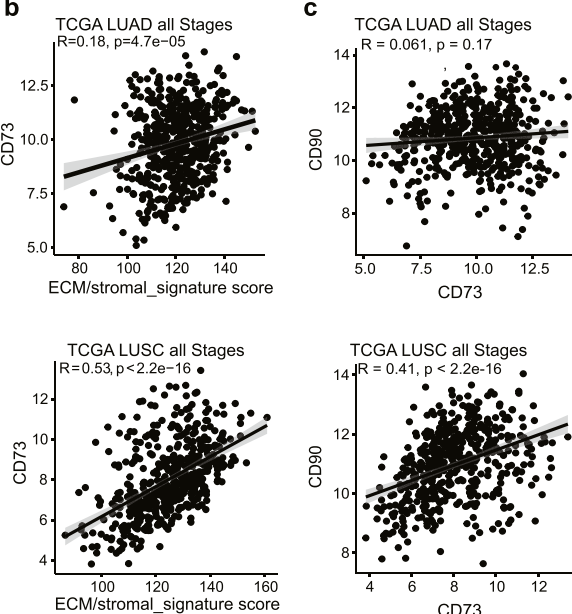

C TCGA LUAD all Stages 14. $R=0.061, p=0.17$
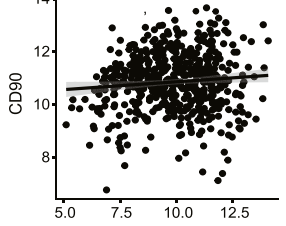
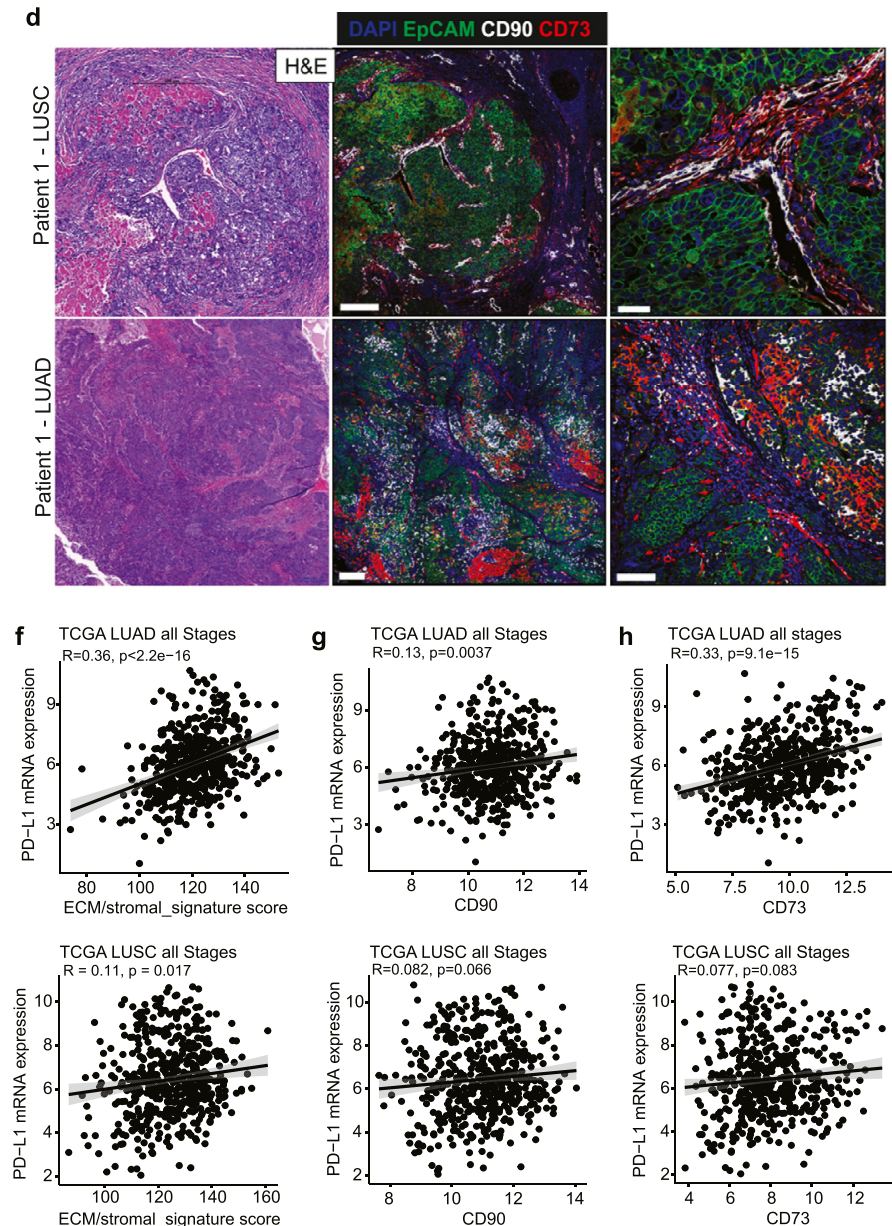

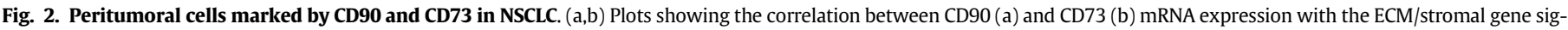

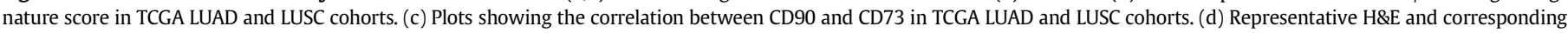

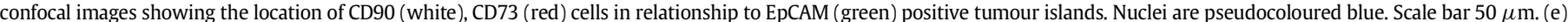

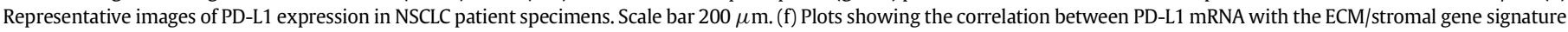
score in TCGA LUAD and LUSC cohorts. (g,h) Plots showing the correlation between PD-L1 with CD90 (g) and CD73 (h) mRNA expression in TCGA LUAD and LUSC cohorts.

in LUAD and LUSC and across the Pan-cancer TGCA cohort (Fig. S3). There was a positive correlation between PD-L1 expression and the $\mathrm{ECM} /$ stromal gene signature in LUAD but not LUSC (Fig. 2f). We also observed a weaker correlation between CD90 and CD73 with PD-L1 in TCGA LUAD, whereas no correlation was observed in LUSC cohorts (Fig. 2g,h). Collectively, these data suggest that CD90 and CD73 cells contained within the nontumor stroma may represent an important contributor to local immune regulation in NSCLC.

\subsection{Expression of immune checkpoint molecules in mesenchymal cell subsets in NSCLC}

To identify the cellular source of ECM/stromal gene signature, we applied our previously developed multiparametric flow cytometric profiling strategy in resected tumours from a larger cohort of human NSCLC patients for the presence of mesenchymal cells based on the expression of CD90 and CD73 and their coexpression of PD-L1 and the innate checkpoint molecule CD47 (Fig. S4a,b) [27]. In tumor (T) and matched uninvolved nontumor $(\mathrm{N})$ digests, the predominant mesenchymal cell subset was CD90-CD73- in both LUAD (N, $76 \% \pm 16 \%$ vs T, $69.8 \% \pm 22 \%$, $\mathrm{p}=0.064)$ and LUSC (N, 73\% $\pm 20 \%$ vs T, $67 \% \pm 26 \%, \mathrm{p}=0.1$ ) (Fig. S4c,d), which expressed low levels of PD-L1 in tumor (Fig. S4c,d). In contrast, PD-L1 overexpression was observed in CD90+CD73+ and CD90+CD73tumor mesenchymal subsets in both $\operatorname{LUAD}(\mathrm{N}, 506 \pm 570$ vs T, 891 \pm $1263, \mathrm{p}<0.0001$; and $\mathrm{N}, 309 \pm 386$ vs T, $580 \pm 1088, \mathrm{p}<0.0001$, respectively) and LUSC (N, 684 \pm 632 vs $\mathrm{T}, 1054 \pm 1237, \mathrm{p}=0.0013$; and $\mathrm{N}$,
$391 \pm 448$ vs T, $596 \pm 979, \mathrm{p}=0.0027$, respectively) (Fig. S4e,f). There was a high negative correlation between \% of CD90-CD73- and CD90 + CD73+ cells in both LUAD and LUSC (Fig. S4g-i).

CD73 together with another cell surface ectonucleotidase CD39 convert extracellular ATP to immunosuppressive adenosine contributing to an immunosuppressive TME [33]. Together with PD-L1, CD73 and CD39 are broadly expressed on the tumor epithelium, stroma and vasculature. To address this further, we used flow cytometry to investigate the expression of these checkpoint molecules in tumor epithelium (EpCAM)_compared with mesenchymal cell susbets and vasculature $(C D 31)$ in a smaller cohort of patients $(n=12)$ (Fig. 3a). Using a sequential gating strategy (Fig. 3b,-d), we showed that $39 \pm 23 \%$ of CD90+CD73+ cells co-expressed CD39 but lacked CD31, whereas $0.6 \pm 1 \%$ of $C D 90+C D 73+$ co-expressed $C D 31$ while lacking CD39 (Fig. 3e). We also found that $19.1 \pm 23.7 \%$ of CD90+CD73 + co-expressed both CD39 and CD31. In comparison, $69 \pm 24 \%$ of the CD90+CD73- mesenchymal subset was found to co-express CD39 while lacking CD31 whereas only $6.2 \pm 7 \%$ of this subset co-expressed both CD39 and CD31. Whereas $28 \pm 27 \%$ of the CD90-CD73+ mesenchymal cell subset co-expressed CD39 only compared with $32.5 \pm 31 \%$ coexpressing both CD39 and CD31. We further observed an upregulation in PD-L1 expression in CD90+CD73+CD39+CD31- compared with CD90+CD73+CD39+CD31+ cells (Fig. 3f, left panel), which was not observed in the CD90+CD73- (Fig. 3f, centre panel) or CD90-CD73+ mesenchymal cell subsets (Fig. 3f, right panel). Moreover, there was a small percentage of mesenchymal cell susbets that co-expressed 

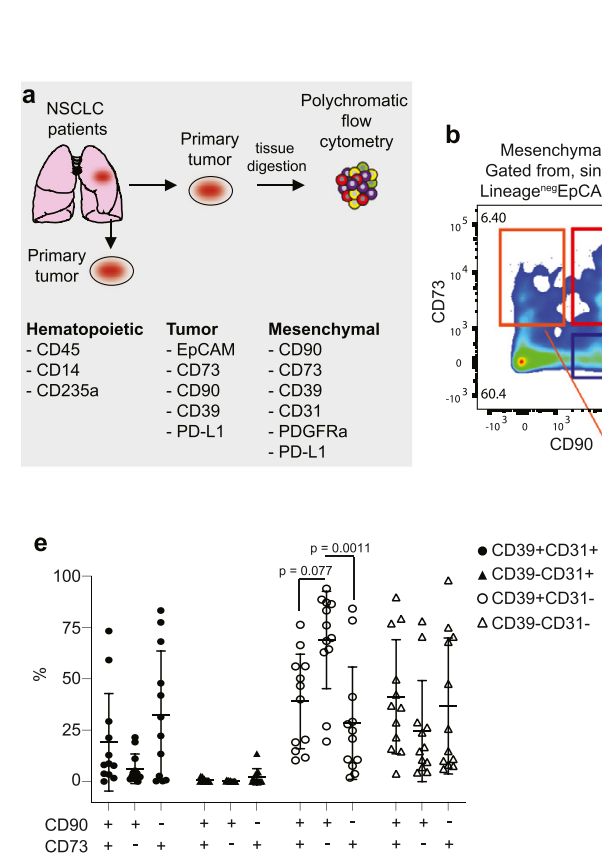

C

d

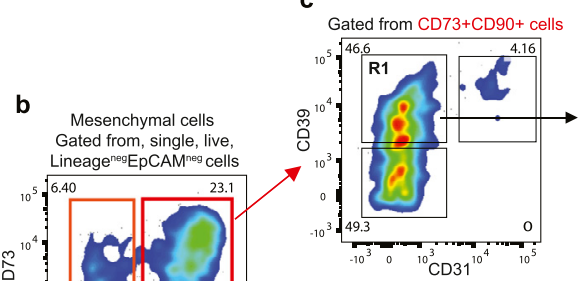

R1 gate, CD39+CD31- cells
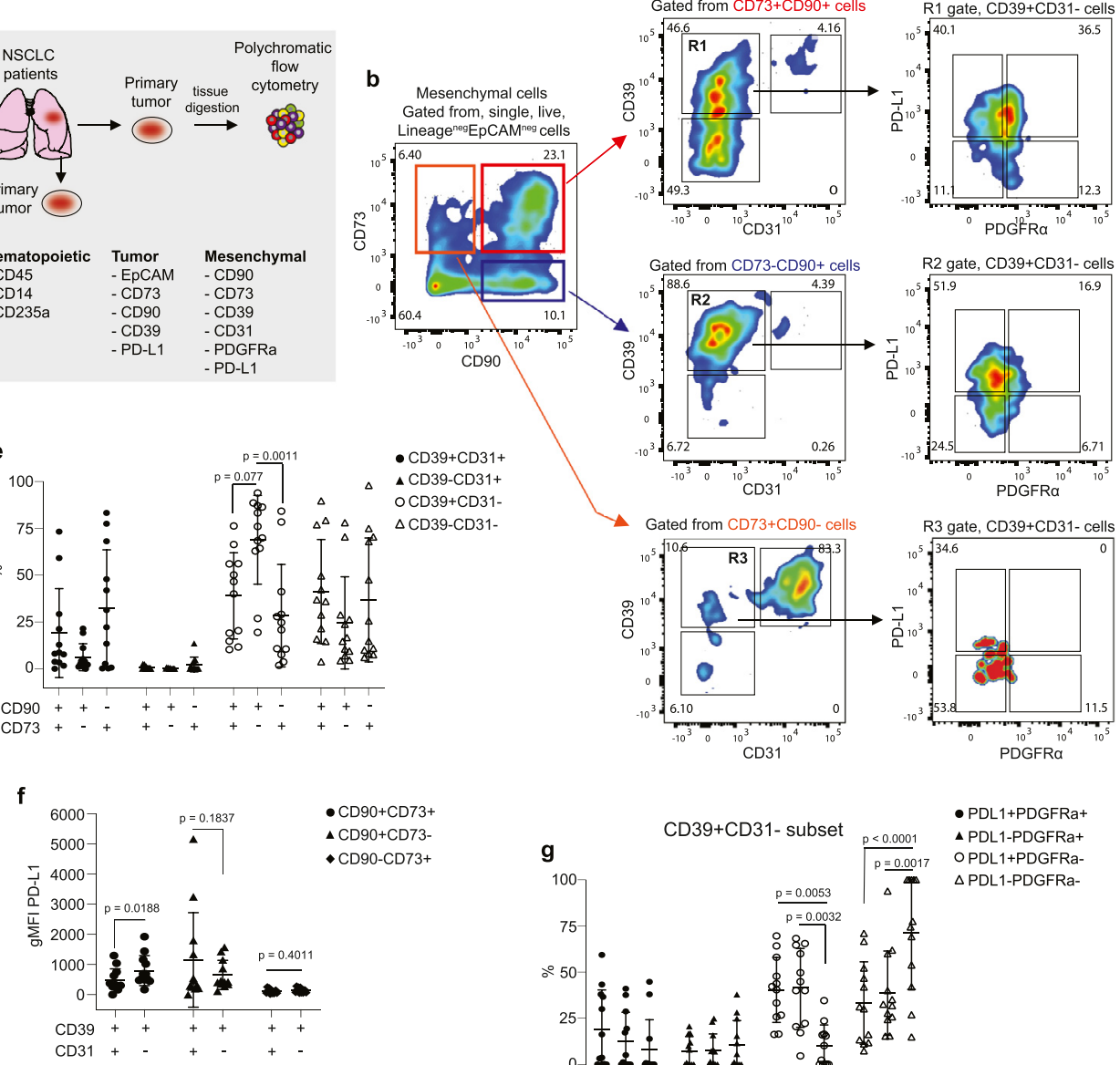

- $\mathrm{CD} 90+\mathrm{CD} 73+$ $\mathrm{CD} 90+\mathrm{CD} 73$

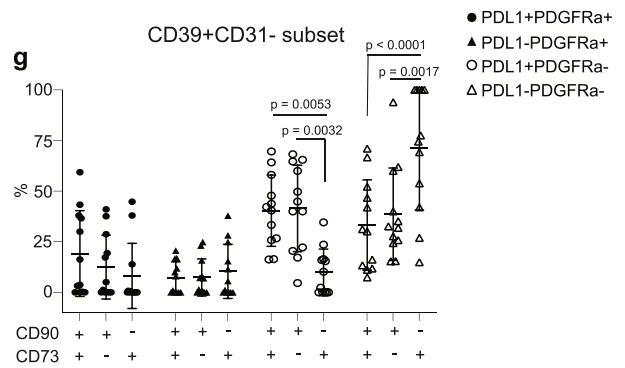

h
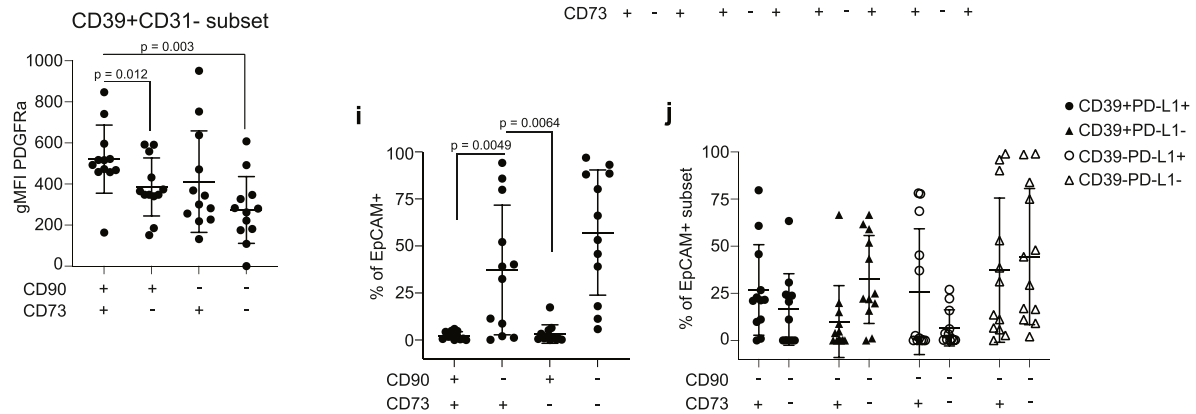

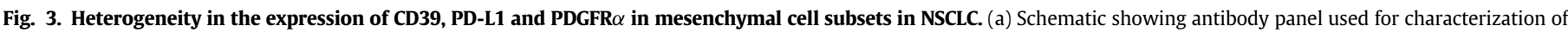

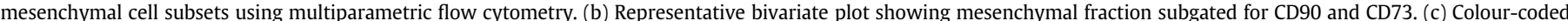

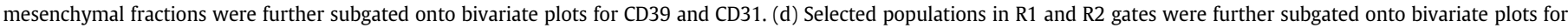

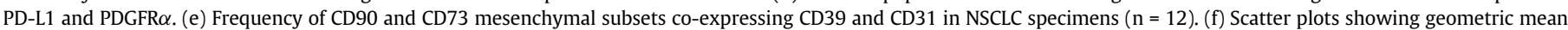

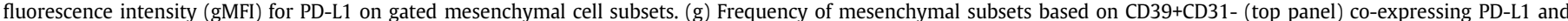

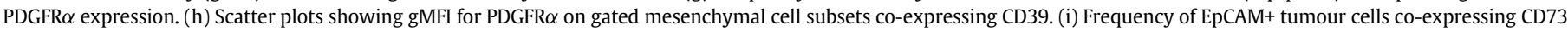

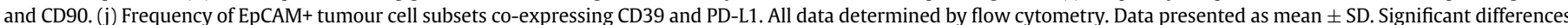

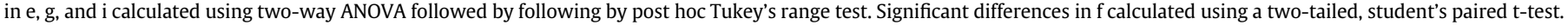

PDGFR $\alpha$ mainly observed in the CD90+CD73+CD39+ and CD90 +CD73-CD39+ cell subsets (Fig. 3g, h). EpCAM+ tumour cells that coexpress CD73 (Fig. S3h) were also found to express CD39 and PD-L1 (Fig. 3i,j). Our data confirm the significant phenotypic heterogeneity in the mesenchymal compartment that has been reported in NSCLC [34].

\section{4. $E C M /$ stromal signature is enriched in peritumoral $C D 90+C D 73+$ mesenchymal cells and amplified by TGF $\beta 1$}

Next, we prospectively isolated the CD90+CD73+ mesenchymal cell subset from both LUAD and LUSC tumour digests and exposed them to the pleiotropic cytokine TGF $\beta 1$ (Fig. 4a). At the mRNA level, genes comprising the $\mathrm{ECM} /$ stromal gene signature were enriched in peritumoral CD90+CD73+ cells and amplified by TGF $\beta 1$ (Fig. 4b). At the protein level, TGF $\beta 1$ upregulated the expression of PDGFR $\beta$ ( $\mathrm{p}<$ 0.05 , paired student's t-test) (Fig. 4c). The same trend was found with PDGFR $\alpha$; however, this did not reach significance. We have previously demonstrated that tumour-associated $\mathrm{CD} 90+\mathrm{CD} 73+$ also express FAP at the mRNA level [27]. Here, TGF $\beta 1$ increased FAP expression in CD90+CD73+ peritumoral cells (Fig. 4b) and FAP+ cells were observed adjacent to and surrounding tumour epithelium (Fig. 4d). To deconvolute the complexity of the mesenchymal compartment, we examined publicly available single-cell RNA sequencing 

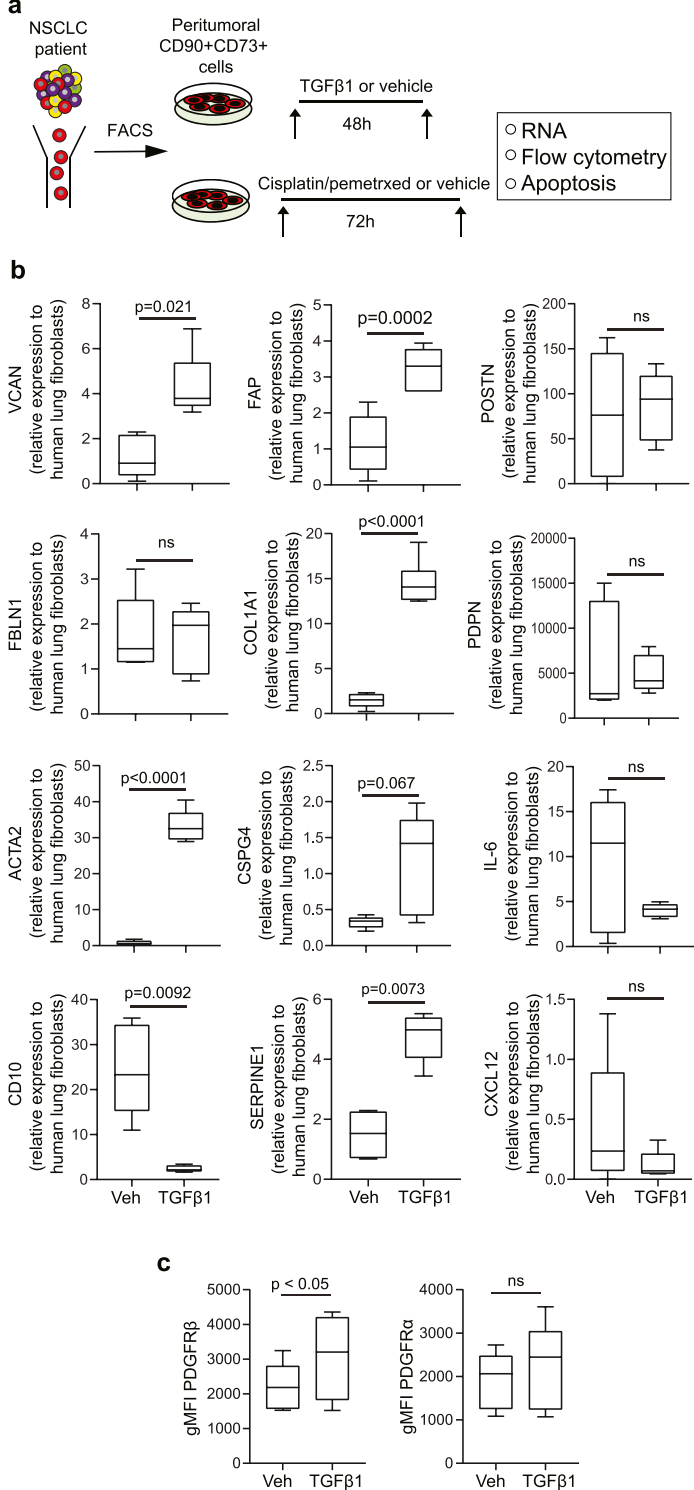

d
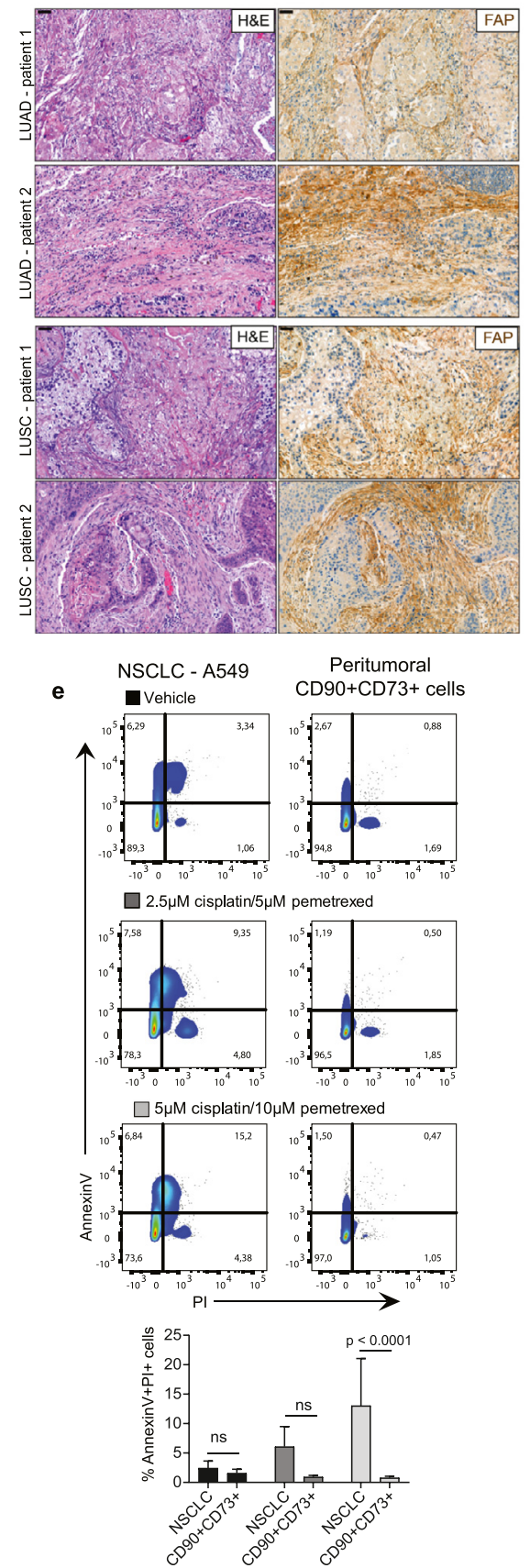

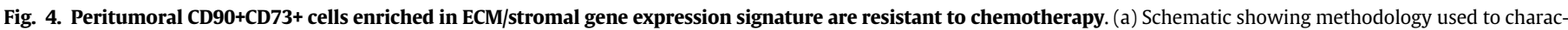

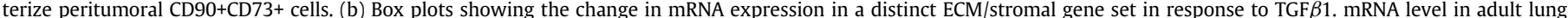

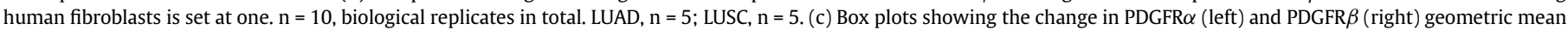

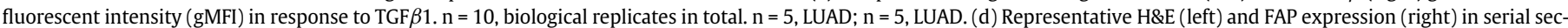

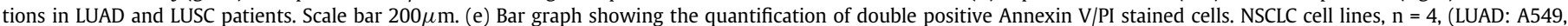

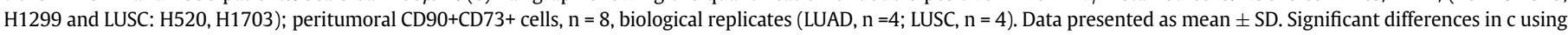
a two-tailed, student's paired t-test. Significant differences in b, e calculated using one way ANOVA following by post hoc Tukey's range test. ns, not significant.

data to validate the mesenchymal marker expression from patients with NSCLC [34] and pancreatic adenocarcinoma (PAAD) [35] downloaded from the Tumor Immune Single-cell Hub [36] to determine the clustering across all cellular compartments. Single-cell analysis reveals enrichment of CD90 (THY1), CD73 (NT5E), and FAP in a cluster of fibroblasts in both NSCLC and PAAD (Fig. S5a,b). In our cohort, 28 patients underwent neoadjuvant chemotherapy prior to surgical resection. Although neoadjuvant chemotherapy had no effect on the mesenchymal cell subset composition or PD-L1 expression compared to non-treated patients (data not shown), treatment of CD90+CD73+ mesenchymal subset with conventional chemotherapy using cisplatin/pemetrexed did not result in a significant increase in Annexin V/PI staining (\% 0.8 \pm 0.3 ) compared with NSCLC cell lines (\% 13.1 \pm 8 ) (Fig. 4e). Collectively, these data suggest that the ECM/stromal gene signature may arise from peritumoral CD90+CD73+ cells, which we previously demonstrated dysregulated perivascular-like function [27] and shown to be the cell of origin of CAFs in well-defined murine models of cancer $[20,21]$. 

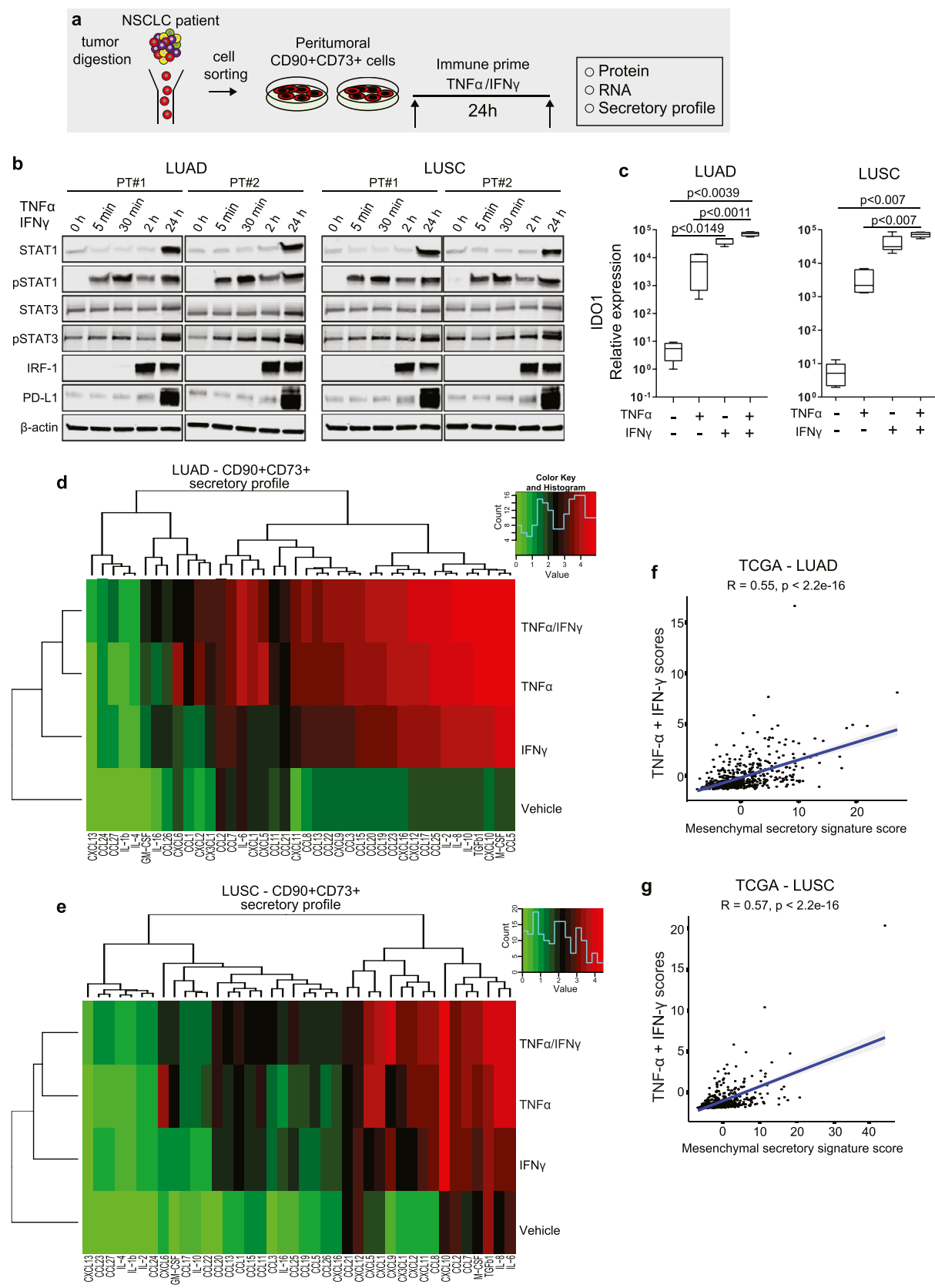

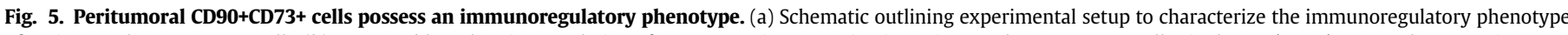

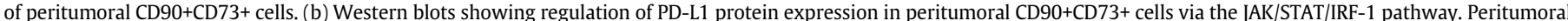

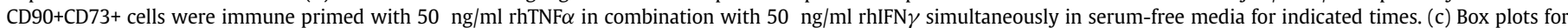

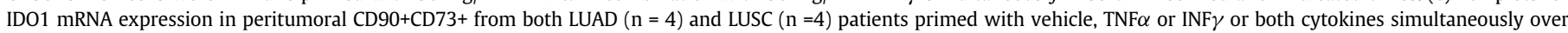

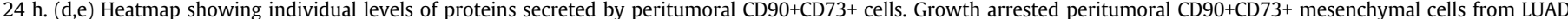

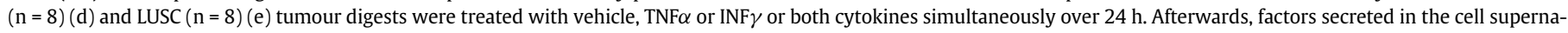

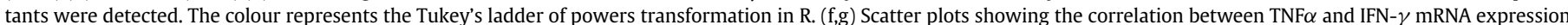

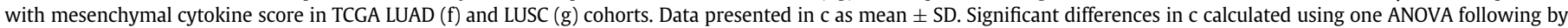
post hoc Tukey's range test.

\subsection{Peritumoral $C D 90+C D 73+$ cells possess an immunoregulatory phenotype}

It is reasonable to postulate that prior to their infiltration into tumour islands, tumour-reactive $\mathrm{T}$ cells would be in direct contact with peritumoral $C D 90+C D 73+$ cells in the stroma. Whether this distinct mesenchymal subset regulates local immune function is unknown. To investigate this, we exposed primary cultures of peritumoral CD90+CD73+ cells (LUSC, $\mathrm{n}=8$; and LUAD, $\mathrm{n}=8$, biological replicates) to TNF $\alpha$ and IFN $\gamma$, two pro-inflammatory cytokines that feature prominently in inflamed tumours (Fig. 5a). Here, PD-L1 was regulated at the protein level by the induction of proteins and phosphorylated proteins involved in the JAK-STAT signalling pathway including STAT1/pSTAT1, STAT3/pSTAT3 and increase expression of IRF-1 (Fig. 5b). IFN $\gamma$ increases the expression of the immunomodulating heme enzyme indoleamine 2, 3 dioxygenase 1 (IDO1), which mediates depletion of tryptophan and suppression of $\mathrm{T}$ cell function [37]. In both primary LUAD- and LUSC-derived peritumoral CD90+CD73+ cells, IDO1 was significantly elevated at the mRNA level following a single treatment with TNF $\alpha$ or IFN $\gamma$ or in combination (Fig. 5c).

To identify factors secreted by CD90+CD73+ peritumoral cells, we performed an analysis of supernatants from immune primed 
peritumoral $\mathrm{CD} 90+\mathrm{CD} 73+$ cells, which revealed a wide range of secreted pro-inflammatory cytokines, $\mathrm{C}-\mathrm{C}$ and $\mathrm{C}-\mathrm{X}-\mathrm{C}$ chemokines and growth factors that are critically involved in the recruitment, retention and functional modification of leukocytes (Fig. 5d,e). Secretion of 15 of 40 analyzed cytokines/chemokines was induced by TNF $\alpha$ only (> 5 fold) and included CCL1, CCL2, CCL5, CCL11, CCL13, CCL20, CCL22, CXCL1, CXCL2, CXCL5, M-CSF, GM-CSF, IL-6, IL-8 and IL-10. Whereas IFN $\gamma$ enhanced the secretion ( $>5$ fold) of IL-16, IL-2, CCL25, CCL2 and CCL23. Combined exposure to TNF $\alpha$ and IFN $\gamma$ resulted in synergistic increases in IL-16, CCL8, CX3CL1, CXCL9 and TGF $\beta 1$, whereas additive effects were found for CCL1, CCL7, CCL15, CCL17, CCL19, CCL24, CCL26, CXCL16, M-CSF and IL-2. TNF $\alpha$ or IFN $\gamma$ alone increased the secretion of CXCL10, CXCL11 and CCL2; however, there was no change when TNF $\alpha$ and IFN $\gamma$ were combined. There were notable differences in the types of cytokines and the amount secreted between LUAD and LUSC peritumoral CD90+CD73+ cells. Based on the top 10 secreted proteins, we generated a mesenchymal secretory signature and found that there was a positive correlation with the expression of TNF $\alpha$ and IFN $\gamma$ in TCGA LUAD and LUSC dataset (Fig. 5f, g). Taken together, these data suggest that peritumoral CD90+CD73+ cells may be part of an important local negative feedback loop that performs immune sentinel function in an attempt to prevent extensive tissue damage while restoring homeostasis following injuryinduced activation of $\mathrm{T}$ cells.

\subsection{Peritumoral $C D 90+C D 73+$ cells suppress $T$ cells}

We next investigated whether peritumoral CD90+CD73+ cells negatively regulate $\mathrm{T}$ cell function. We performed in vitro functional assays using CFSE-labelled CD3+ T cells derived from blood of healthy donors stimulated with staphylococcus enterotoxin B (SEB) (Fig. 6a). We chose the microbial protein SEB to activate $T$ cells based on its function as a superantigen and immune activator inducing strong $\mathrm{T}$ cell stimulation via binding directly to $\mathrm{T}$ cell receptor (TCR) and MHC-II receptor [38]. Using co-culture conditions, peritumoral CD90 +CD73+ cells from both LUSC and LUAD specimens suppressed SEBinduced proliferation of CFSE-labeled CD3+ T cells at a 1:1 and 5:1 ratio ( $\mathrm{T}$ cells: $\mathrm{CD} 90+\mathrm{CD} 73+$ cells), irrespective of immune priming with TNF $\alpha /$ IFN $\gamma$ (Fig. 6b,c). Supernatants (SN) from IP peritumoral CD90+CD73+ cells also effectively blocked the proliferation of SEBactivated T cells (Fig. $6 \mathrm{c}$ ). The potent inhibition was confirmed using a transwell culture system (Fig. 6d,e). PD-L1 was upregulated in peritumoral CD90+CD73+ mesenchymal cells at a ratio of 1:1 (Fig. 6f), which was associated with a decrease in the expression of the activation markers PD-1 and CD107a, as well as the homeostatic regulator CD127 (Fig. 6g). These phenotypic changes coincided with suppression in the release of cytotoxic cytokines TNF $\alpha$ and IFN $\gamma$ (Fig. 6h). Together, these data show that peritumoral CD90+CD73+ cells are naturally equipped with an immune regulatory function. Therefore, in a highly inflamed TME, peritumoral CD90+CD73+ mesenchymal cells may restrict host antitumor $\mathrm{T}$ cell responses in an adaptive manner resulting in local immunosuppression.

\subsection{Neutralization of PD-L1 and TFG $\beta 1$ combined with IDO1 inhibition in peritumoral $C D 90+C D 73+$ cells relieves $T$ cell immune suppression}

Next, we investigated the functional consequences of peritumoral CD90+CD73+ cells on tumour-infiltrating lymphocytes (TILs) isolated from patient tumour material (Fig. 7a). Peritumoral CD90+CD73+ cells abrogated the proliferation of SEB-activated CFSE-labeled CD3+ TILs (Fig. 7b,c). Cocultures treated with neutralizing antibodies against PD-L1 or chemical inhibition of IDO1 alone resulted in marginal changes in proliferation and no change in expression of CD127, a key homeostatic regulator of $\mathrm{T}$ cell survival and proliferation via IL7 signalling [39]. However, combined neutralization of PD-L1 and TGF $\beta 1$ with inhibition of IDO1 resulted in the restoration of TIL proliferation while increasing expression of CD127 (Fig. 7b-d). In contrast, combined neutralization of PD-L1 and TGF $\beta 1$ only, or neutralization of PD-L1 with IDO1 inhibition or neutralization of TGF $\beta 1$ with ID01 inhibition only did not restore TIL proliferation (Fig. S7a, b). We also found that pretreatment of peritumoral CD90+CD73+ cells with $\alpha, \beta$-Methyleneadenosine $5^{\prime}$-diphosphate (AMP-CP), an inhibitor of CD73 nucleoside activity, also failed to restore TIL function (Fig. S6a,b). In separate experiments, we isolated and expanded $\mathrm{T}$ cells from the uninvolved lung tissue. In cocultures of uninvolved lung-derived $T$ cells activated with SEB with peritumoral CD90+CD73 + cells, we demonstrate that combined neutralization of PD-L1 and TGF $\beta 1$ with inhibition of ID01 also restored degranulation (CD107a) and IFN $\gamma$ secretion of CD8+ (Fig. 7e,f) and CD4+ T cells (Fig. 7g,h). In contrast, this was not observed after pretreating peritumoral CD90 + CD73+ cells with combined blockade of PD-L1 with TGF $\beta 1$, PD-L1 with IDO1 inhibition or TGF $\beta 1$ with IDO1 inhibition or AMP-CP (Fig. S6c,d). Collectively, these data suggest that peritumoral CD90+CD73+ cells may serve a critical role in shaping $\mathrm{T}$ cell function in NSCLC. More importantly, our findings highlight the importance of blocking PD-L1 in combination with independent biological features of the mesenchymal compartment in the tumour stroma.

\section{Discussion}

Since the prognostic and predictive role of PD-L1 staining in solid tumours remains uncertain [40], significant attention also has been placed on the abundance and localization of CD8+ T effector cells as a better prognostic and predictive biomarker due to their association with improved patient outcomes [16]. In support of this, an IFN $\gamma$ driven T-cell inflamed gene expression profile is associated with improved response to PD-1 blockade with pembrolizumab across multiple tumour types [22]. Using a core set of genes indicative of a CD8+ T cell effector phenotype, we observed a high enrichment of this IA signature score in NSCLC across the TCGA Pan-cancer cohort. These immunologically active tumours are enriched in a core set of genes defining an ECM/stromal signature. Importantly, a higher ECM/stromal to IA gene signature ratio is associated with poor survival. We further show that in primary resected NSCLC tumours, peritumoral cells that coexpress CD90 and CD73 are enriched in genes comprising the ECM/ stromal gene signature, which were enhanced by TGF $\beta 1$. Peritumoral CD90+CD73+ cells found in both LUAD and LUSC samples can prevent the physiological activation of $\mathrm{T}$ cells using both cell contact and soluble mediators. Importantly, only combined PD-L1 and TGF $\beta 1$ blockade together with IDO1 inhibition was able to restore T cell function.

There is a growing body of evidence to support the TGF $\beta$-responsive non-hematopoietic stromal compartment in regulating host antitumor immunity and response to ICB in solid tumours in humans [17-19,41]. In human metastatic urothelial cancer, TGF $\beta$-primed peritumoral fibroblasts function to exclude CD8+ TILs limiting the response to PD-L1 blockade using atezolizumab [18]. The pan-fibroblast $\operatorname{TGF} \beta$ response signature was highest in inflamed tumours or tumours where T cells were localized within the stroma. Using the EMT6 murine tumour model that recapitulates this immuneexcluded tumour phenotype, combined blockade of PD-L1 and TGF $\beta$ reprogrammed peritumoral fibroblasts converted tumours from an excluded to inflamed phenotype resulting in a reduction in tumour burden [18]. TGF $\beta$-primed stroma has been implicated in the exclusion of TILs in a murine model of metastatic colon cancer [41]. In support of this, a stroma-mediated source of immune resistance and lack of response to PD-1 blockade was reported in a separate cohort of patients with urothelial cancer [19]. In both LUAD and LUSC tumour digests, not only are peritumoral CD90+CD73+ cells enriched in the $\mathrm{ECM} /$ stromal gene signature, which was amplified by $\operatorname{TGF} \beta 1$, but these cells themselves are a major source of inflammatory-driven TGF $\beta 1$ secretion. Besides TGF $\beta 1$, inflammatory-driven CD90+CD73+ peritumoral cells secrete a wide range of cytokines belonging to the 
a

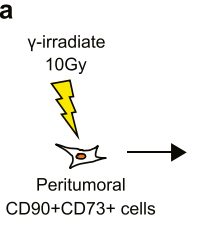

b

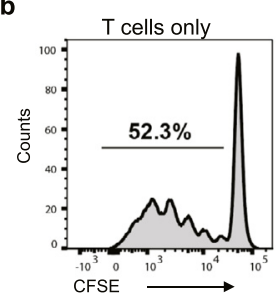

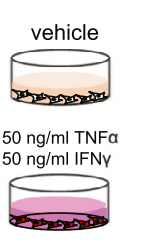

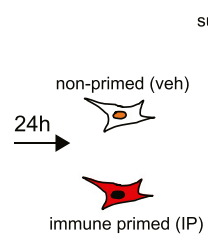

Collect

$(\mathrm{SN})$

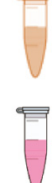

SEB activated $\mathrm{SE}+\mathrm{CD} 3+\mathrm{T}$ cells O

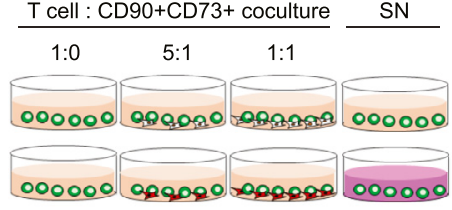

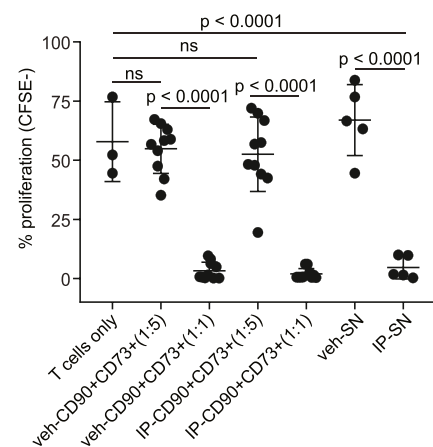
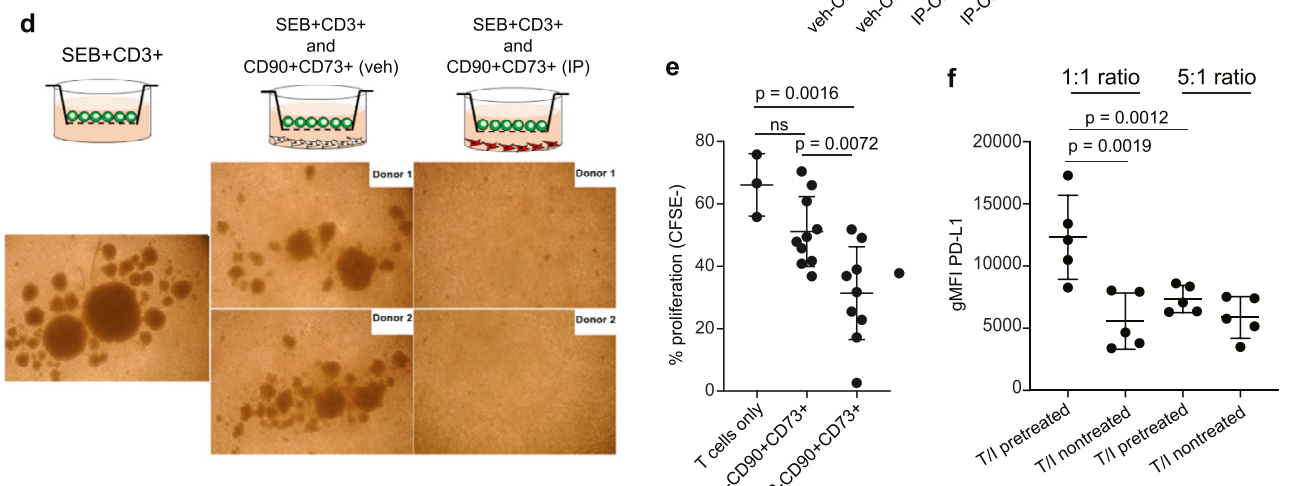

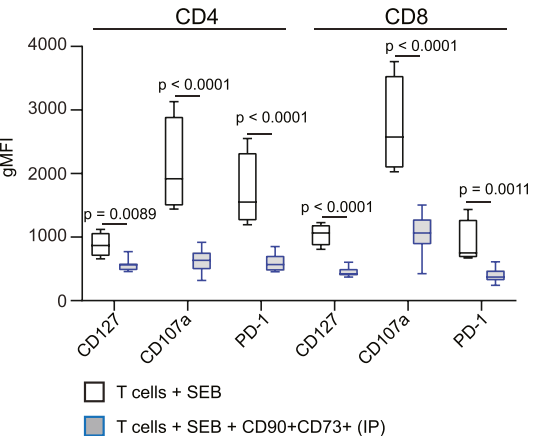

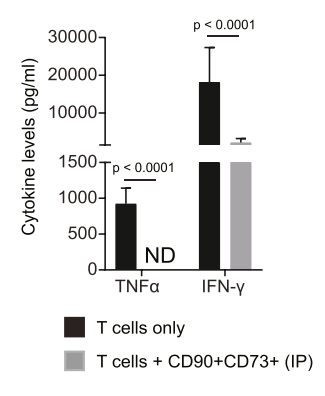

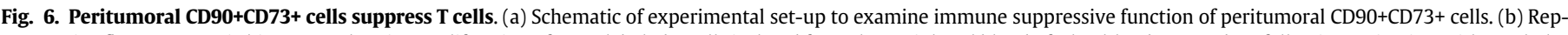

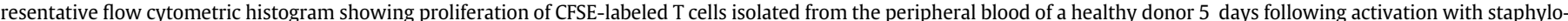

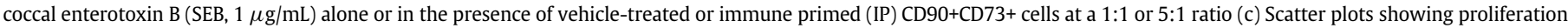

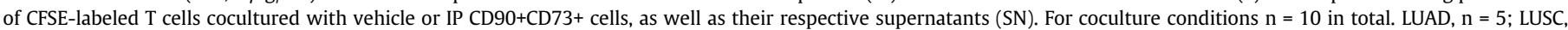

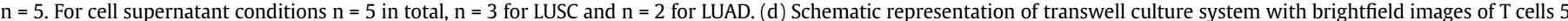

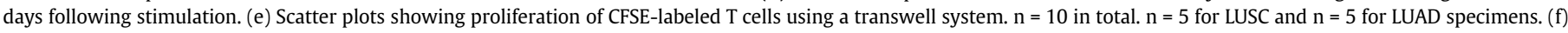

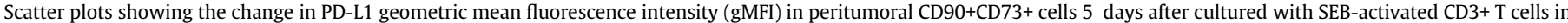

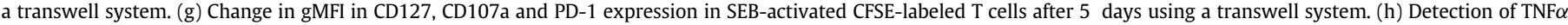

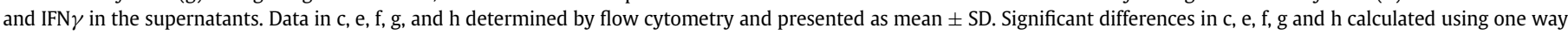
ANOVA following by post hoc Tukey's range test. ns, not significant.

$\mathrm{C}-\mathrm{C}$ and $\mathrm{C}-\mathrm{X}-\mathrm{C}$ chemokine family that are chemotactic for immune cells and also contribute to wound healing. The most abundant $\mathrm{C}-\mathrm{X}-\mathrm{C}$ chemokines secreted were CXCL9 and CXCL10, which are critical regulators of immune cell recruitment/activation via the CXCR3 axis in T cells, as well as NK cells [42]. This paracrine axis could serve to attract and retain $T$ cells in the stroma. As shown in fresh ex vivo slices of human lung tumours, the dense stroma acts as a physical barrier preventing $T$ cells from infiltrating tumour islands, which was most prominent in the perivascular region [14]. Moreover, CD90-positive mesenchymal stromal cells from human LUSC suppress NK cell cytotoxic function via the release of soluble prostaglandin E2 (PGE2) [43]. The comparability between the mesenchymal populations is unclear, as plastic adherence was used in the aforementioned study compared with the prospective isolation of a discrete subpopulation of tumor-associated mesenchymal cella using a FACS-based approach in our study. Nonetheless, CD90-positive mesenchymal cells in both the perivascular and stromal regions of human lung NSCLC correlate with poor patient prognosis $[43,44]$.

Surprisingly, our study reveals that TNF $\alpha$ is a more potent driver of the stromal immune regulatory secretome than IFN $\gamma$. We could 


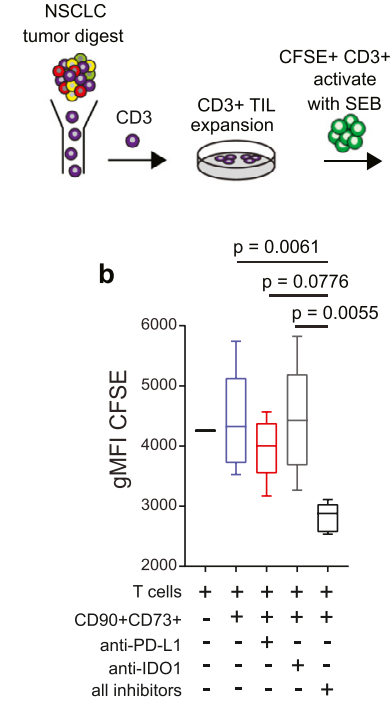

Transwell culture CD3+ TILs:CD90+CD73+ cells
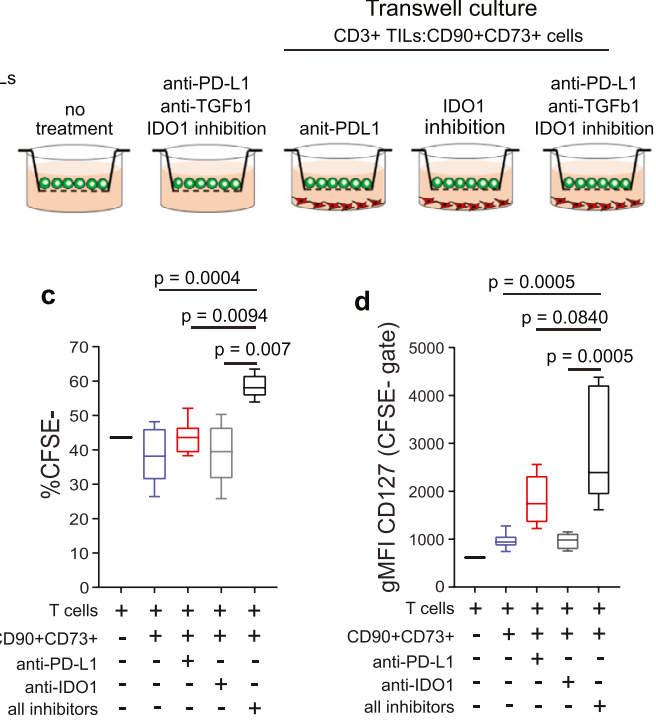

e
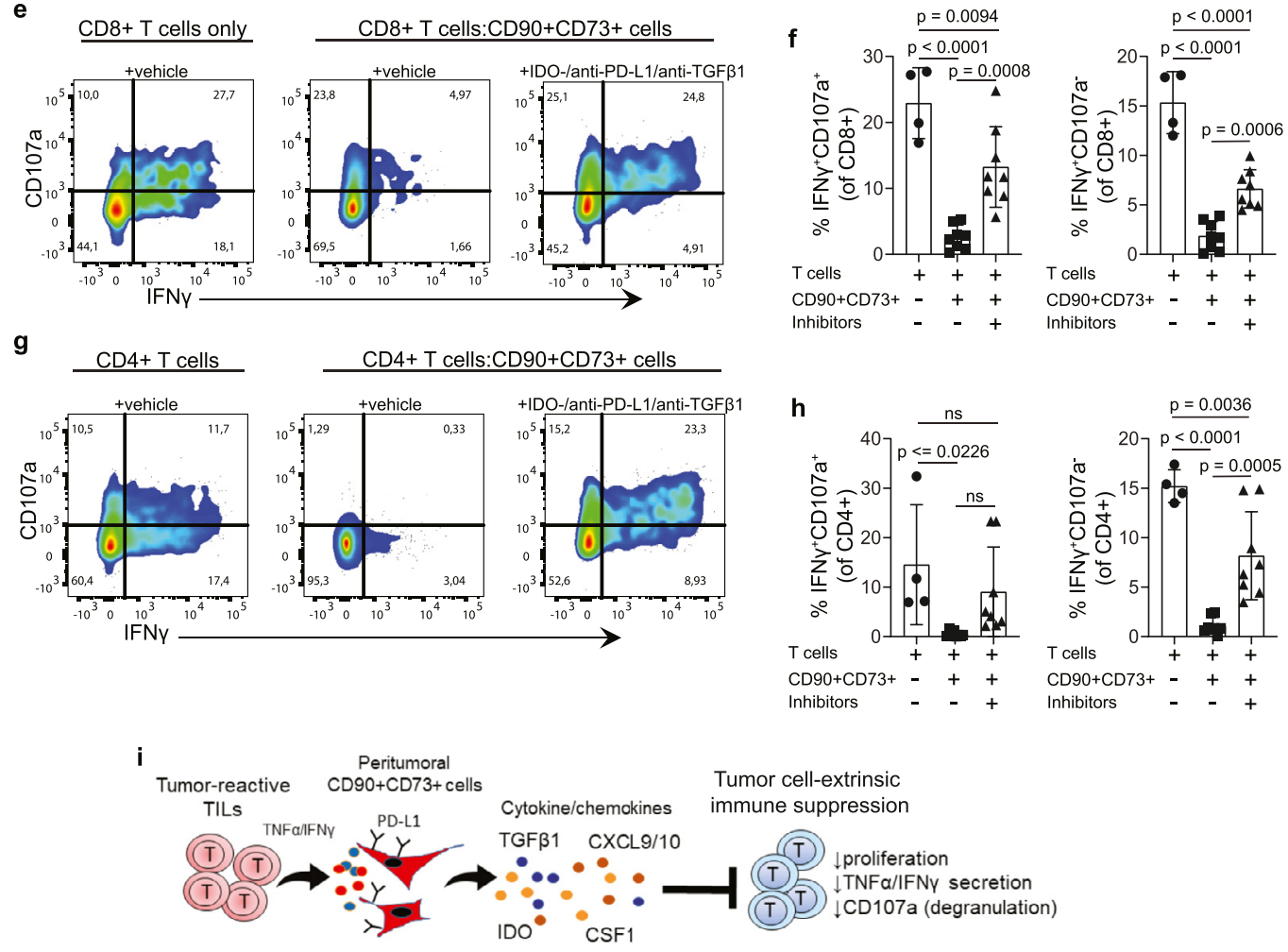

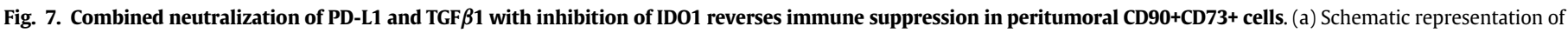

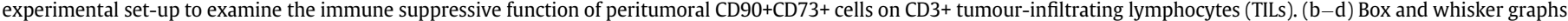

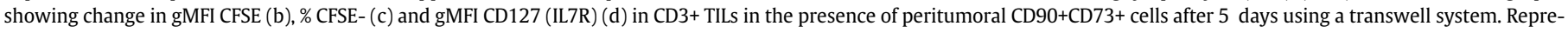

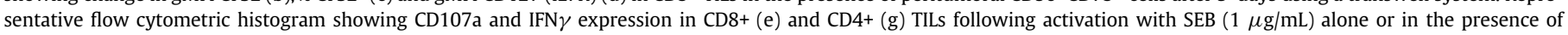

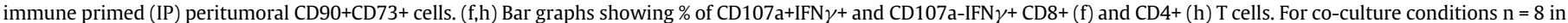

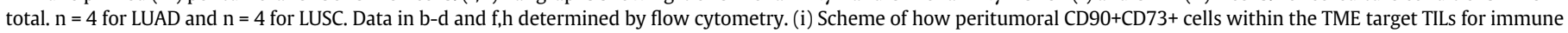
suppression. Data presented as mean \pm SD. Significant differences in b, c, d, f and h calculated using one way ANOVA following by post hoc Tukey's range test. ns, not significant.

only reverse the suppressive function of peritumoral CD90+CD73+ mesenchymal cells by a combined blockade of PD-L1, TGF $\beta 1$ and IDO1 but not by using them as single agents. In human breast cancer, isolation of a FAP+PDGFR $\beta+$ CAF subset was shown to promote survival and differentiation of inhibitory Tregs via both a cell contact and paracrine mechanism involving CXCL12 [45] and was extended to mesenchymal high-grade serous ovarian cancers [46]. Recently, a population of $\mathrm{CD} 90+$ mesenchymal cells found in human and mouse breast tumours contained subsets of FAP+PDPN+ and FAP+PDPN- cells with tumor growth-promoting properties; however, only the CD90+FAP+PDPN+ subset was immune suppressive [47]. Recently, single-cell analysis of human breast tumours identified a cluster of FAP+ fibroblasts whereby subpopulations enriched in ECM proteins and TGF $\beta 1$ signalling were linked with regulation of host immune response via upregulation of PD-1 and CTLA4 expression on Tregs [48]. Importantly, these subclusters were further shown to be enriched in patients with metastatic melanoma and NSCLC that failed to respond to ICB compared with responders. 
Here, our findings highlight ways by which a highly inflamed TME may drive the transition of peritumoral CD90+CD73+ cells into critical regulators of $\mathrm{T}$ effector cell function and provide a potential means of targeting this axis to boost host antitumor immunity and response to ICB in the setting of NSCLC. However, our study has several limitations. First, we did not assess whether the relationship between IA and ECM/ stromal signature scores on patient outcomes is associated with neoantigen burden and whether this differs based on histological subtype. In LUAD, tumours with a high clonal neoantigen burden are more homogenous and associated with improved response to ICB [49]. Homogenous tumours with a high neoantigen burden are marked by an inflamed TME with significant upregulation of PD-L1 and IL-6 [50]. This was not observed in LUSC, which are more heterogeneous [50]. Homogeneous LUAD tumours were also enriched in genes associated with a CD8+ T cell effector phenotype. Based on our analysis, the association between $\mathrm{PD}-\mathrm{L} 1$ and the ECM/stromal gene signature was stronger in LUAD compared with LUSC. Moreover, IL-6 represented the most prominent cytokine secreted from both LUAD and LUSC-derived CD90+CD73+ peritumoral cells in response to TNF $\alpha$. A comprehensive molecular analysis across several solid cancer subtypes supports a TGF $\beta$-responsive stromal signature as an independent predictor of failure to ICB, irrespective of tumour mutational burden [17]. Although our prelimary data show that simultaneous blockade of PD-L1, IDO and TGF $\beta 1$ restores, in part, TIL function, a deeper immunogenomic profiling is required to gain a greater understanding of the molecular underpinnings driving this response. Therefore, future studies require linking multifactorial cellular and molecular profiling together with neoantigen burden to identify subsets of patients that may benefit from immune checkpoint blockade combined with targeting independent biological features of the tumour stroma in NSCLC. Secondly, we were not able to address the contextual cues from cancer cells that drive the stromal directives. Human lung CD90+CD73+ cells support de novo vessel formation [25,26], resembling pericyte-like support cells [51]. However, this capability becomes dyregualted in tumor-associated CD90+CD73+ cells [27]. Here, TGF $\beta 1$ upregulates $\operatorname{PDGFR} \beta$ expression in peritumoral CD90 $+C D 73+$ cells, which is invovled in the recruitment of pericytes to growing tumours via tumor-derived PDGF-BB [21]. Therefore, whether $\operatorname{PDGFR} \beta /$ PDGF-BB axis is invovled in the initial recruitment of resident CD90+CD73+ cells to growing tumors requires further investigation in a well-defined murine tumour model. Thirdly, while PD1/PD-L1 and CTLA4 are important immune checkpoints, the immunosuppressive adenosine molecule in the tumour stroma represents an emerging therapeutic target [52]. Adenosine is derived from the breakdown of extracellular ATP coming from dying cancer cells and other sources as a result of hypoxia and celluar stress via two ectonucleotidases CD39 and CD73, which we show are co-expressed by peritumoral mesenchymal cells that also co-express PD-L1. We were not able to determine the contribution of these two ectonucleotidases to the immunosuppressive function of CD90+CD73+ peritumoral cells, as targeting CD73 alone was without any benefit. Despite this, the CD73/adenosine pathway is upregulated in both treatment naïve and resitant EGFR-mutant NSCLC, which is associated with features of immune suppression [53]. While antiCD73 therapy boosts the efficacy of anti-PD-L1 and anti-CTLA4 ICB in murine tumour models [54], it is not clear whether additional targeting of CD39 would offer any additional benefit in NSCLC [33]. Finally, the significance of blocking the enzymatic breakdown of intratumoral ATP causing rising intratumoural levels of eATP would depend heavily on intratumoral cell types present and their ability to uptake eATP together with expression levels of purinergic receptors [55]. eATP can have direct immune stimulatory effects on several target immune cell populations including dendritic cells (DC) enhancing chemotaxis and maturation via eATP binding to the P2Y2 and P2X purinergic receptors [55]. This would also offset the immunosuppressive function of eADO on DC maturation, thereby having a beneficial impact on the adaptive anti-tumor immune response. That said, the boost in eATP-dependent immunostimulation might be offset by a pro-tumorigenic effect of eATP directly on cancer cells. Intratumoral eATP binds to purinergic receptors such as P2X purinergic receptor 7 (P2RX7) located on malignant cells, which has been shown in melanoma [56]. High intratumoral eATP would stimulate cancer cell metabolism in P2 $\times 7 \mathrm{R}$ positive cancer cells, which leads to a depletion of nutrients, acidification of the TME and recruitment of myeloid-derived suppressor cells that together would culminate in T cell anergy. Chen and colleagues [57] demonstrate that cancer cells also internalize eATP via macropinocytosis, providing a much-needed energy source fueling cellular growth, metabolism and migration, which has been reported from both melanoma and NSCLC [58]. Thus, removal of ATP from the extracellular space involving ATP consumption by highly proliferating lung cancer cells could potentially be more advantageous, as this would promote cancer cell metabolism via enhanced glucose flux satisfying the increase in energetic and biosynthetic demands [59]. Moving forward, preclinical validation in intact organisms with a competent immune system will be required to determine whether there is a causal link between targeting features of peritumoral CD90 $+\mathrm{CD} 73+$ cells and enhanced host antitumor immunity and response to ICB in NSCLC $[17,48]$. Second, it may be necessary to test these combinatorial ICB together with strategies that target ATP/purinergic signaling with P2X antagonists on tumor growth and the potential for serious side effects.

\section{Author contributions}

LW - assisted in study design, data collection, data analysis, data interpretation, created figures, edited manuscript

HY - assisted in data collection, data analysis, data interpretation, created figures and edited manuscript

PD - assisted in data collection, data analysis and edited manuscript

SB - assisted in data collection, data analysis, data interpretation and edited manuscript

FB - assisted with data collection and edited manuscript

$\mathrm{CW}$ - assisted with data collection and edited manuscript

TMM - assisted with data collection and edited manuscript

$\mathrm{RP}$ - assisted with data collection and edited manuscript

$\mathrm{NH}$ - assisted with data collection and edited manuscript

WS - assisted with data collection and edited manuscript

RAS - assisted with data collection, funding and edited manuscript

SRRH - study design, data collection, data analysis, data interpretation, created figures, wrote and edited manuscript

\section{Declaration of Competing Interest}

The authors declare no potential conflicts of interest

\section{Acknowledgments}

The authors acknowledge the technical support from the Translational Research Unit of the Institute of Pathology in performing immunohistochemical staining. The fresh tissue samples were provided via the Tissue Bank Bern, Institute of Pathology, University of Bern. We also acknowledge technical support for FACS provided by members of the University of Bern FACSLab.

\section{Data sharing statement}

Data in this study are available upon reasonable request from the corresponding author at srrhall@protonmail.com.

\section{Supplementary materials}

Supplementary material associated with this article can be found in the online version at doi:10.1016/j.ebiom.2021.103664. 


\section{References}

[1] Brahmer JR, Tykodi SS, Chow LQ, Hwu WJ, Topalian SL, Hwu P, et al. Safety and activity of anti-PD-L1 antibody in patients with advanced cancer. N Engl J Med 2012:366(26):2455-65.

[2] Garon EB, Hellmann MD, Rizvi NA, Carcereny E, Leighl NB, Ahn MJ, et al. Five-year overall survival for patients with advanced nonsmall-cell lung cancer treated with pembrolizumab: results from the phase I KEYNOTE-001 study. J Clin Oncol 2019;37(28):2518-27

[3] Peters S, Reck M, Smit EF, Mok T, Hellmann MD. How to make the best use of immunotherapy as first-line treatment for advanced/metastatic non-small-cell lung cancer. Ann Oncol 2019;30(6):884-96.

[4] Reck M, Rodriguez-Abreu D, Robinson AG, Hui R, Csoszi T, Fulop A, et al. Pembrolizumab versus chemotherapy for PD-L1-positive non-small-cell lung cancer. N Engl J Med 2016;375(19):1823-33.

[5] Socinski MA, Jotte RM, Cappuzzo F, Orlandi F, Stroyakovskiy D, Nogami N, et al. Atezolizumab for first-line treatment of metastatic nonsquamous NSCLC. N Engl Med 2018;378(24):2288-301.

[6] Doroshow DB, Sanmamed MF, Hastings K, Politi K, Rimm DL, Chen L, et al. Immunotherapy in non-small cell lung cancer: facts and hopes. Clin Cancer Res. 2019;25 (15):4592-602 an official journal of the American Association for Cancer Research.

[7] Liu R, Merola J, Manes TD, Oin L, Tietjen GT, Lopez-Giraldez F, et al. Interferongamma converts human microvascular pericytes into negative regulators of alloimmunity through induction of indoleamine 2,3-dioxygenase 1. JCI Insight 2018;3(5):e97881.

[8] Binnewies M, Roberts EW, Kersten K, Chan V, Fearon DF, Merad M, et al. Understanding the tumor immune microenvironment (TIME) for effective therapy. Nat Med 2018;24(5):541-50.

[9] Ivashkiv LB. IFNgamma: signalling, epigenetics and roles in immunity, metabolism, disease and cancer immunotherapy. Nat Rev Immunol 2018;18(9):545-58.

[10] Chen Z, Fillmore CM, Hammerman PS, Kim CF, Wong KK. Non-small-cell lung cancers: a heterogeneous set of diseases. Nat RevCancer 2014;14(8):535-46.

[11] Egeblad M, Nakasone ES, Werb Z. Tumors as organs: complex tissues that interface with the entire organism. Dev Cell 2010;18(6):884-901.

[12] Giraldo NA, Sanchez-Salas R, Peske JD, Vano Y, Becht E, Petitprez F, et al. The clinical role of the TME in solid cancer. Br J Cancer 2019;120(1):45-53.

[13] Kalluri R. The biology and function of fibroblasts in cancer. Nat Rev Cancer 2016;16(9):582-98.

[14] Salmon H, Franciszkiewicz K, Damotte D, Dieu-Nosjean MC, Validire P, Trautmann A et al. Matrix architecture defines the preferential localization and migration of T cells into the stroma of human lung tumors. J Clin Investig 2012;122(3):899-910.

[15] Tirosh I, Izar B, Prakadan SM, Wadsworth MH, 2nd TD, Trombetta JJ, et al. Dissecting the multicellular ecosystem of metastatic melanoma by single-cell RNA-seq. Science 2016;352(6282):189-96.

[16] Galon J, Costes A, Sanchez-Cabo F, Kirilovsky A, Mlecnik B, Lagorce-Pages C, et al Type, density, and location of immune cells within human colorectal tumors predict clinical outcome. Science 2006:313(5795):1960-4

[17] Chakravarthy A, Khan L, Bensler NP, Bose P, De Carvalho DD. TGF-beta-associated extracellular matrix genes link cancer-associated fibroblasts to immune evasion and immunotherapy failure. Nat Commun 2018:9(1):4692.

[18] Mariathasan S, Turley SJ, Nickles D, Castiglioni A, Yuen K, Wang Y, et al. TGFbeta attenuates tumour response to PD-L1 blockade by contributing to exclusion of T cells. Nature 2018;554(7693):544-8.

[19] Wang L, Saci A, Szabo PM, Chasalow SD, Castillo-Martin M, Domingo-Domenech J, et al. EMT- and stroma-related gene expression and resistance to PD-1 blockade in urothelial cancer. Nat Commun 2018;9(1):3503.

[20] Arina A, Idel C, Hyjek EM, Alegre ML, Wang Y, Bindokas VP, et al. Tumor-associated fibroblasts predominantly come from local and not circulating precursors. Proc Natl Acad Sci USA 2016;113(27):7551-6.

[21] Hosaka K, Yang Y, Seki T, Fischer C, Dubey O, Fredlund E, et al. Pericyte-fibroblast transition promotes tumor growth and metastasis. Proc Natl Acad Sci USA 2016;113(38):E5618-27

[22] Ayers M, Lunceford J, Nebozhyn M, Murphy E, Loboda A, Kaufman DR, et al. IFNgamma-related mRNA profile predicts clinical response to PD-1 blockade. J Clin Investig 2017;127(8):2930-40.

[23] Guo X, Zhang Y, Zheng L, Zheng C, Song J, Zhang Q et al. Global characterization of T cells in non-small-cell lung cancer by single-cell sequencing. Nat Med 2018;24 (7):978-85.

[24] Rooney MS, Shukla SA, Wu CJ, Getz G, Hacohen N. Molecular and genetic properties of tumors associated with local immune cytolytic activity. Cell 2015;160(1-2):48-61.

[25] Bichsel CA, Hall SR, Schmid RA, Guenat OT, Geiser T. Primary human lung pericytes support and stabilize in vitro perfusable microvessels. Tissue Eng Part A 2015;21(15-16):2166-76

[26] Wang L, Dorn P, Zeinali S, Froment L, Berezowska S, Kocher GJ, et al. CD90(+) CD146(+) identifies a pulmonary mesenchymal cell subtype with both immune modulatory and perivascular-like function in postnatal human lung. Am J Physiol Lung Cell Mol Physiol 2020;318(4):L813-L30.

[27] Bichsel CA, Wang L, Froment L, Berezowska S, Muller S, Dorn P, et al. Increased PD-L1 expression and IL-6 secretion characterize human lung tumor-derived perivascular-like cells that promote vascular leakage in a perfusable microvasculature model. Sci Rep 2017;7(1):10636.

[28] Kowanetz M, Zou W, Gettinger SN, Koeppen H, Kockx M, Schmid P, et al. Differential regulation of PD-L1 expression by immune and tumor cells in NSCLC and the response to treatment with atezolizumab (anti-PD-L1). Proc Natl Acad Sci USA 2018;115(43):E10119-E26.
[29] Prelaj A, Tay R, Ferrara R, Chaput N, Besse B, Califano R. Predictive biomarkers of response for immune checkpoint inhibitors in non-small-cell lung cancer. Eur J Cancer 2019;106:144-59.

[30] Galon J, Bruni D. Approaches to treat immune hot, altered and cold tumours with combination immunotherapies. Nat Rev Drug Discov 2019;18(3):197-218.

[31] Saltz J, Gupta R, Hou L, Kurc T, Singh P, Nguyen V, et al. Spatial organization and molecular correlation of tumor-infiltrating lymphocytes using deep learning on pathology images. Cell Rep 2018;23(1):181-93 e7.

[32] Thorsson V, Gibbs DL, Brown SD, Wolf D, Bortone DS, Ou Yang TH, et al. The immune landscape of cancer. Immunity 2018;48(4):812-30 e14.

[33] Li XY, Moesta AK, Xiao C, Nakamura K, Casey M, Zhang H, et al. Targeting CD39 in cancer reveals an extracellular ATP- and inflammasome-driven tumor immunity, Cancer Discov 2019;9(12):1754-73.

[34] Lambrechts D, Wauters E, Boeckx B, Aibar S, Nittner D, Burton O, et al. Phenotype molding of stromal cells in the lung tumor microenvironment. Nat Med 2018;24 (8):1277-89.

[35] Helms E, Onate MK, Sherman MH. Fibroblast heterogeneity in the pancreatic tumor microenvironment. Cancer Discov 2020;10(5):648-56.

[36] Sun D, Wang J, Han Y, Dong X, Ge J, Zheng R, et al. TISCH: a comprehensive web resource enabling interactive single-cell transcriptome visualization of tumor microenvironment. Nucleic Acids Res 2021;49(D1):D1420-D30.

[37] Spranger S, Spaapen RM, Zha Y, Williams J, Meng Y, Ha TT, et al. Up-regulation of PD-L1, IDO, and T(regs) in the melanoma tumor microenvironment is driven by CD8(+) T cells. Sci Transl Med 2013:5(200):200ra116.

[38] Kappler J, Kotzin B, Herron L, Gelfand EW, Bigler RD, Boylston A, et al. V beta-specific stimulation of human T cells by staphylococcal toxins. Science 1989;244 (4906):811-3.

[39] Saini M, Pearson C, Seddon B. Regulation of T cell-dendritic cell interactions by IL7 governs T-cell activation and homeostasis. Blood 2009;113(23):5793-800

[40] Nishino M, Ramaiya NH, Hatabu H, Hodi FS. Monitoring immune-checkpoint blockade: response evaluation and biomarker development. Nat Rev Clin Oncol 2017;14(11):655-68.

[41] Tauriello DVF, Palomo-Ponce S, Stork D, Berenguer-Llergo A, Badia-Ramentol J, Iglesias $\mathrm{M}$, et al. TGFbeta drives immune evasion in genetically reconstituted colon cancer metastasis. Nature 2018;554(7693):538-43.

[42] Tokunaga R, Zhang W, Naseem M, Puccini A, Berger MD, Soni S, et al. CXCL9, CXCL10, CXCL11/CXCR3 axis for immune activation-a target for novel cancer therapy. Cancer Treat Rev 2018:63:40-7.

[43] Galland S, Vuille J, Martin P, Letovanec I, Caignard A, Fregni G, et al. Tumorderived mesenchymal stem cells use distinct mechanisms to block the activity of natural killer cell subsets. Cell Rep 2017;20(12):2891-905.

[44] Schliekelman MJ, Creighton CJ, Baird BN, Chen Y, Banerjee P, Bota-Rabassedas N, et al. Thy-1(+) cancer-associated fibroblasts adversely impact lung cancer prognosis. Sci Rep 2017;7(1):6478.

[45] Costa A, Kieffer Y, Scholer-Dahirel A, Pelon F, Bourachot B, Cardon M, et al. Fibroblast heterogeneity and immunosuppressive environment in human breast cancer. Cancer Cell 2018;33(3):463-79 e10.

[46] Givel AM, Kieffer Y, Scholer-Dahirel A, Sirven P, Cardon M, Pelon F, et al, miR200regulated CXCL12beta promotes fibroblast heterogeneity and immunosuppression in ovarian cancers. Nat Commun 2018;9(1):1056

[47] Cremasco V, Astarita JL, Grauel AL, Keerthivasan S, MacIsaac K, Woodruff MC, et al. FAP delineates heterogeneous and functionally divergent stromal cells in immune-excluded breast tumors. Cancer Immunol Res 2018;6(12):1472-85.

[48] Kieffer Y, Hocine HR, Gentric G, Pelon F, Bernard C, Bourachot B, et al. Single-cell analysis reveals fibroblast clusters linked to immunotherapy resistance in cancer. Cancer Discov 2020;10(9):1330-51.

[49] Rizvi NA, Hellmann MD, Snyder A, Kvistborg P, Makarov V, Havel JJ, et al. Cancer immunology. Mutational landscape determines sensitivity to PD-1 blockade in non-small cell lung cancer. Science 2015·348(6230):124-8.

[50] McGranahan N, Furness AJ, Rosenthal R, Ramskov S, Lyngaa R, Saini SK, et al. Clonal neoantigens elicit $\mathrm{T}$ cell immunoreactivity and sensitivity to immune checkpoint blockade. Science 2016;351(6280):1463-9.

[51] Weibel ER. On pericytes, particularly their existence on lung capillaries. Microvasc Res 1974;8(2):218-35.

[52] Boison D, Yegutkin GG. Adenosine metabolism: emerging concepts for cancer therapy. Cancer Cell 2019:36(6):582-96.

[53] Le X, Negrao MV, Reuben A, Federico L, Diao L, McGrail D, et al. Characterization of the immune landscape of EGFR-mutant NSCLC identifies CD73/adenosine pathway as a potential therapeutic target. J Thorac Oncol 2021;16(4):583-600.

[54] Roh M, Wainwright DA, Wu JD, Wan Y, Zhang B. Targeting CD73 to augment cancer immunotherapy. Curr Opin Pharmacol 2020;53:66-76.

[55] Kepp O, Bezu L, Yamazaki T, Di Virgilio F, Smyth MJ, Kroemer G, et al. ATP and cancer immunosurveillance. EMBO J 2021:40(13):e108130.

[56] Martin S, Dudek-Peric AM, Garg AD, Roose H, Demirsoy S, Van Eygen S, et al. An autophagy-driven pathway of ATP secretion supports the aggressive phenotype of $\mathrm{BRAF}(\mathrm{V} 600 \mathrm{E})$ inhibitor-resistant metastatic melanoma cells. Autophagy 2017;13(9):1512-27.

[57] Qian Y, Wang X, Liu Y, Li Y, Colvin RA, Tong L, et al. Extracellular ATP is internalized by macropinocytosis and induces intracellular ATP increase and drug resistance in cancer cells. Cancer Lett 2014;351(2):242-51.

[58] Qian Y, Wang X, Li Y, Cao Y, Chen X. Extracellular ATP a new player in cancer metabolism: NSCLC cells internalize ATP in vitro and in vivo using multiple endocytic mechanisms. Mol Cancer Res 2016;14(11):1087-96.

[59] Israelsen WJ, Vander Heiden MG. ATP consumption promotes cancer metabolism. Cell 2010;143(5):669-71. 